\title{
Estimating change points in nonparametric time series regression models
}

\author{
Maria Mohr ${ }^{1} \cdot$ Leonie Selk ${ }^{1}$
}

Received: 16 September 2019 / Revised: 13 January 2020 / Published online: 27 January 2020

(c) The Author(s) 2020

\begin{abstract}
In this paper we consider a regression model that allows for time series covariates as well as heteroscedasticity with a regression function that is modelled nonparametrically. We assume that the regression function changes at some unknown time $\left\lfloor n s_{0}\right\rfloor$, $s_{0} \in(0,1)$, and our aim is to estimate the (rescaled) change point $s_{0}$. The considered estimator is based on a Kolmogorov-Smirnov functional of the marked empirical process of residuals. We show consistency of the estimator and prove a rate of convergence of $O_{P}\left(n^{-1}\right)$ which in this case is clearly optimal as there are only $n$ points in the sequence. Additionally we investigate the case of lagged dependent covariates, that is, autoregression models with a change in the nonparametric (auto-) regression function and give a consistency result. The method of proof also allows for different kinds of functionals such that Cramér-von Mises type estimators can be considered similarly. The approach extends existing literature by allowing nonparametric models, time series data as well as heteroscedasticity. Finite sample simulations indicate the good performance of our estimator in regression as well as autoregression models and a real data example shows its applicability in practise.
\end{abstract}

Keywords Change point estimation - Time series · Nonparametric regression · Autoregression · Conditional heteroscedasticity · Consistency · Rates of convergence

Mathematics Subject Classification Primary 62G05; Secondary 62G08 - 62G20 . $62 \mathrm{M} 10$

\section{Introduction}

Change point analysis has gained attention for decades in mathematical statistics. There is a vast literature on testing for structural breaks when the possible timing of

Leonie Selk

leonie.selk@math.uni-hamburg.de

1 Department of Mathematics, University of Hamburg, Hamburg, Germany 
such a break, the change point, is unknown, see for instance Kirch and Kamgaing (2012) and reference mentioned therein. This paper, however, is concerned with the estimation of the change point when assuming its existence.

The most simple set of models can be described as follows

$$
Y_{t}=\mu_{1} I\left\{t \leq\left\lfloor n s_{0}\right\rfloor\right\}+\mu_{2} I\left\{t>\left\lfloor n s_{0}\right\rfloor\right\}+\varepsilon_{t}, t=1, \ldots, n
$$

where $s_{0} \in(0,1)$ is the (rescaled) change point, $\mu_{1}$ and $\mu_{2}$ the signal before and after the break, respectively, and $\left(\varepsilon_{t}\right)_{t}$ being stationary and centred errors. These models are often referred to as AMOC-models (at most one change). The problem naturally moved from the standard case with independent errors (see Ferger and Stute (1992) among others) to the time series context. Both Bai (1994) and Antoch et al. (1997) allow for linear processes and Hušková and Kirch (2008) more generally for dependent errors.

Additional information on the form of the signal can be expressed through a process of covariates $\left(X_{t}\right)_{t}$ resulting in linear regression models with a change in the regression parameter, such as

$$
Y_{t}=\beta_{1} X_{t} I\left\{t \leq\left\lfloor n s_{0}\right\rfloor\right\}+\beta_{2} X_{t} I\left\{t>\left\lfloor n s_{0}\right\rfloor\right\}+\varepsilon_{t}, t=1, \ldots, n,
$$

where $\beta_{1}$ and $\beta_{2}$ are the regression coefficients before and after the break, respectively. Bai (1997), Horváth et al. (1997) and Aue et al. (2012) among others consider the estimation of a change point in (multiple) linear regression models making use of least squares estimation. Considering $X_{t}=Y_{t-1}$ in the linear regression model from above, one obtains autoregressive models with one change in the autoregressive parameter. The estimation of the parameters and the unknown change point in $\operatorname{AR}(1)$ models was for instance considered by Chong (2001), Pang et al. (2014) and Pang and Zhang (2015).

Our aim is to propose an estimator for the change point $s_{0}$ in a nonparametric version of the regression model from above, namely

$$
Y_{t}=m_{(1)}\left(X_{t}\right) I\left\{t \leq\left\lfloor n s_{0}\right\rfloor\right\}+m_{(2)}\left(X_{t}\right) I\left\{t>\left\lfloor n s_{0}\right\rfloor\right\}+\varepsilon_{t}, t=1, \ldots, n,
$$

for some nonparametric regression functions $m_{(1)}, m_{(2)}$ (before and after the break) and in addition also investigate the autoregressive case where $X_{t}=Y_{t-1}$. While the investigation of points of discontinuity in (nonparametric) regression functions has been studied to some extend (see for instance Döring and Jensen (2015) for an overview), not that much research has been devoted to change point analysis in nonparametric models as the one above, where the change occurs in time. Delgado and Hidalgo (2000) propose estimators for the location and size of structural breaks in a nonparametric regression model imposing scalar breaks in time or values taken by some regressors, as in threshold models. Their rates of convergence and limiting distribution depends on a bandwidth, chosen for the kernel estimation. Chen et al. (2005) estimate the time of a scalar change in the conditional variance function in nonparametric heteroscedastic regression models using a hybrid procedure that combines the least squares and nonparametric methods. 
The paper at hand extends existing literature, on the one hand by allowing for nonparametric heteroscedastic regression models with a general change in the unknown regression function where both errors and covariates are allowed to be time series, and on the other hand by investigating the autoregressive case. The achieved rate of convergence for the proposed estimator of $O_{P}\left(n^{-1}\right)$ is optimal as described in Hariz et al. (2007).

The remainder of the paper is organized as follows. The model and the considered estimator are introduced in Sect. 2. Section 3 contains the regularity assumptions as well as the asymptotic results for the proposed estimator. Section 4 is concerned with the special case of lagged dependent covariates, that is the autoregressive case. In Sect. 5 we describe a simulation study and discuss a real data example, whereas Sect. 6 concludes the paper. Proofs of the main results as well as auxiliary lemmata can be found in the Appendix.

\section{The model and estimator}

Let $\left\{\left(Y_{t}, \boldsymbol{X}_{t}\right): t \in \mathbb{N}\right\}$ be a weakly dependent stochastic process in $\mathbb{R} \times \mathbb{R}^{d}$ following the regression model

$$
Y_{t}=m_{t}\left(\boldsymbol{X}_{t}\right)+U_{t}, t \in \mathbb{N} \text {. }
$$

The unobservable innovations are assumed to fulfill $E\left[U_{t} \mid \mathcal{F}^{t}\right]=0$ almost surely for the sigma-field $\mathcal{F}^{t}=\sigma\left(U_{j-1}, \boldsymbol{X}_{j}: j \leq t\right)$. We assume there exists a change point in the regression function such that

$$
m_{n, t}(\cdot)=m_{t}(\cdot)=\left\{\begin{array}{ll}
m_{(1)}(\cdot), & t=1, \ldots,\left\lfloor n s_{0}\right\rfloor \\
m_{(2)}(\cdot), & t=\left\lfloor n s_{0}\right\rfloor+1, \ldots, n
\end{array}, \quad m_{(1)} \not \equiv m_{(2)}\right.
$$

where $\left\lfloor n s_{0}\right\rfloor$ with $s_{0} \in(0,1)$ is the unknown time the change occurs. Note that we keep above notations for simplicity reasons, however, the considered process is in fact a triangular array process $\left\{\left(Y_{n, t}, \boldsymbol{X}_{n, t}\right): 1 \leq t \leq n, n \in \mathbb{N}\right\}$ and will be treated appropriately.

Assuming $\left(Y_{1}, \boldsymbol{X}_{1}\right), \ldots,\left(Y_{n}, \boldsymbol{X}_{n}\right)$ have been observed, the aim is to estimate $s_{0}$. The idea is to base the estimator on the sequential marked empirical process of residuals, namely

$$
\hat{T}_{n}(s, z):=\frac{1}{n} \sum_{i=1}^{\lfloor n s\rfloor}\left(Y_{i}-\hat{m}_{n}\left(\boldsymbol{X}_{i}\right)\right) \omega_{n}\left(\boldsymbol{X}_{i}\right) I\left\{\boldsymbol{X}_{i} \leq z\right\}
$$

for $s \in[0,1]$ and $z \in \mathbb{R}^{d}$, where $\boldsymbol{x} \leq \boldsymbol{y}$ is short for $x_{j} \leq y_{j}$ for all $j=1, \ldots, d$, $\omega_{n}(\cdot)=I\left\{\cdot \in J_{n}\right\}$ being from assumption (V) below and $\hat{m}_{n}$ being the Nadaraya- 
Watson estimator, that is

$$
\hat{m}_{n}(\boldsymbol{x})=\frac{\sum_{j=1}^{n} K\left(\frac{\boldsymbol{x}-\boldsymbol{X}_{j}}{h_{n}}\right) Y_{j}}{\sum_{j=1}^{n} K\left(\frac{\boldsymbol{x}-\boldsymbol{X}_{j}}{h_{n}}\right)},
$$

with kernel function $K$ and bandwidth $h_{n}$ as considered in the assumptions below. Then we want to estimate $s_{0}$ by

$$
\hat{s}_{n}:=\min \left\{s: \sup _{z \in \mathbb{R}^{d}}\left|\hat{T}_{n}(s, z)\right|=\sup _{\bar{s} \in[0,1]} \sup _{z \in \mathbb{R}^{d}}\left|\hat{T}_{n}(\bar{s}, z)\right|\right\} .
$$

Note that $\hat{s}_{n}=\left\lfloor n \hat{s}_{n}\right\rfloor / n$.

Remark The advantage of using marked residuals in comparison to using the classical CUSUM $\hat{T}_{n}(s, \infty)$ to estimate the change point is that in the first case the estimator is consistent for all changes of the form (2.2) whereas there are several examples in which the use of $\hat{T}_{n}(s, \infty)$ leads to a non-consistent estimator. To this end see the remark below the proof of Theorem 3.1 and compare to Mohr and Neumeyer (2019).

Remark Mohr and Neumeyer (2019) constructed procedures based on functionals of $\hat{T}_{n}$, e.g. a Kolmogorov-Smirnov test statistic $\sup _{s \in[0,1]} \sup _{z \in \mathbb{R}^{d}}\left|\hat{T}_{n}(s, z)\right|$, to test the null hypothesis of no changes in the unknown regression function against change point alternatives as in (2.2). Given that such a test has rejected the null, the use of an M-estimator as in (2.3) seems natural. Furthermore, Cramér-von Mises type test statistics of the form $\sup _{s \in[0,1]} \int_{\mathbb{R}^{d}}\left|\hat{T}_{n}(s, z)\right|^{2} v(z) d z$ for some integrable $v: \mathbb{R}^{d} \rightarrow \mathbb{R}$ were also considered by Mohr and Neumeyer (2019). Assuming strict stationarity of the covariates and the existence of a density $f$ such that $\boldsymbol{X}_{t} \sim f$ for all $t$, as in (IX.1) below, the Cramér-von Mises approach from above with $v \equiv f$ leads to an alternative estimator for $s_{0}$, namely

$\tilde{s}_{n}:=\min \left\{s:\left(\int_{\mathbb{R}^{d}}\left|\hat{T}_{n}(s, z)\right|^{2} f(z) d z\right)^{1 / 2}=\sup _{\bar{s} \in[0,1]}\left(\int_{\mathbb{R}^{d}}\left|\hat{T}_{n}(\bar{s}, z)\right|^{2} f(z) d z\right)^{1 / 2}\right\}$.

However, to obtain a feasible estimator one needs to replace the integral $\int_{\mathbb{R}^{d}}\left|\hat{T}_{n}(s, z)\right|^{2}$ $f(z) d z$ by its empirical counterpart $\frac{1}{n} \sum_{k=1}^{n}\left|\hat{T}_{n}\left(s, \boldsymbol{X}_{k}\right)\right|^{2}$ in practise as $f$ is not known.

\section{Asymptotic results}

In this section we will derive asymptotic properties for $\hat{s}_{n}$. To this end we introduce the following assumptions.

(I) For all $t \in \mathbb{Z}$ let $E\left[U_{t} \mid \mathcal{F}^{t}\right]=0$ a.s. for $\mathcal{F}^{t}=\sigma\left(U_{j-1}, \boldsymbol{X}_{j}: j \leq t\right)$ and $E\left[\left|U_{t}\right|^{q}\right] \leq C_{U}$ for some $C_{U}<\infty$ and $q>2$. 
(II) For all $t \in \mathbb{Z}$ let $E\left[\left|m_{(1)}\left(\boldsymbol{X}_{t}\right)-m_{(2)}\left(\boldsymbol{X}_{t}\right)\right|^{r}\right] \leq C_{m}$ for some $C_{m}<\infty$ and $r>2$.

(III) Let $\left\{\left(Y_{t}, X_{t}\right): 1 \leq t \leq n, n \in \mathbb{N}\right\}$ be strongly mixing with mixing coefficient $\alpha(\cdot)$. For $q, r$ from assumptions (I) and (II) and $b:=\min (q, r)$ let $\alpha(t)=$ $O\left(t^{-\bar{\alpha}}\right)$ with some $\bar{\alpha}>\max ((1+(b-1)(1+d)) /(b-2),(b+2) /(b-2))$.

(IV) For $b$ from assumption (III) let $E\left[\left|Y_{t}\right|^{b}\right]<\infty$ and let $\boldsymbol{X}_{t}$ be absolutely continuous with density function $f_{t}: \mathbb{R}^{d} \rightarrow \mathbb{R}$ that satisfies $\sup _{\boldsymbol{x} \in \mathbb{R}^{d}} E\left[\left|Y_{t}\right|^{b} \mid \boldsymbol{X}_{t}=\right.$ $\boldsymbol{x}] f_{t}(\boldsymbol{x})<\infty$ and $\sup _{\boldsymbol{x} \in \mathbb{R}^{d}} f_{t}(\boldsymbol{x})<\infty$ for all $t \in\{1, \ldots, n\}$ and $n \in \mathbb{N}$. Let there exist some $L \geq 0$ such that $\sup _{|i-j| \geq L} \sup _{\boldsymbol{x}_{i}, \boldsymbol{x}_{j}} E\left[\left|Y_{i} Y_{j}\right| \mid \boldsymbol{X}_{i}=\boldsymbol{x}_{i}, \boldsymbol{X}_{j}=\right.$ $\left.\boldsymbol{x}_{j}\right] f_{i j}\left(\boldsymbol{x}_{i}, \boldsymbol{x}_{j}\right)<\infty$ for all $n \in \mathbb{N}$, where $f_{i j}$ is the density function of $\left(\boldsymbol{X}_{i}, \boldsymbol{X}_{j}\right)$.

(V) Let $\left(c_{n}\right)_{n \in \mathbb{N}}$ be a positive sequence of real valued numbers satisfying $c_{n} \rightarrow \infty$ and $c_{n}=O\left((\log n)^{1 / d}\right)$ and let $\boldsymbol{J}_{n}=\left[-c_{n}, c_{n}\right]^{d}$.

(VI) For some $C<\infty$ and $c_{n}$ from assumption (V) let $\boldsymbol{I}_{n}=\left[-c_{n}-C h_{n}, c_{n}+\right.$ $\left.C h_{n}\right]^{d}$ and let $\delta_{n}^{-1}=\inf _{\boldsymbol{x} \in \boldsymbol{J}_{n}} \inf _{1 \leq t \leq n} f_{t}(\boldsymbol{x})>0$ for all $n \in \mathbb{N}$. Further, let for all $n \in \mathbb{N}$

$$
\begin{aligned}
p_{n} & =\max _{|\boldsymbol{k}|=1} \sup _{\boldsymbol{x} \in \boldsymbol{I}_{n}} \sup _{1 \leq t \leq n}\left|D^{\boldsymbol{k}} f_{t}(\boldsymbol{x})\right|<\infty \\
0<q_{n} & =\max _{0 \leq|\boldsymbol{k}| \leq 1} \sup _{\boldsymbol{x} \in \boldsymbol{I}_{n}} \max _{j=1,2}\left|D^{k} m_{(j)}(\boldsymbol{x})\right|<\infty,
\end{aligned}
$$

where $|\boldsymbol{i}|=\sum_{j=1}^{d} i_{j}$ and $D^{i}=\frac{\partial^{|i|}}{\partial x_{1}^{i_{1}} \ldots \partial x_{d}^{i_{d}}}$ for $\boldsymbol{i}=\left(i_{1}, \ldots, i_{d}\right) \in \mathbb{N}_{0}^{d}$.

(VII) Let $K: \mathbb{R}^{d} \rightarrow \mathbb{R}$ be symmetric in each component with $\int_{\mathbb{R}^{d}} K(z) d z=1$ and compact support $[-C, C]^{d}$. Additionally let $|K(\boldsymbol{u})|<\infty$ for all $\boldsymbol{u} \in \mathbb{R}^{d}$ and $\left|K(\boldsymbol{u})-K\left(\boldsymbol{u}^{\prime}\right)\right| \leq \Lambda\left\|\boldsymbol{u}-\boldsymbol{u}^{\prime}\right\|$ for some $\Lambda<\infty$ and for all $\boldsymbol{u}, \boldsymbol{u}^{\prime} \in \mathbb{R}^{d}$, where $\|\boldsymbol{x}\|=\max _{i=1, \ldots, d}\left|x_{i}\right|$.

(VIII) With $b$ and $\bar{\alpha}$ from assumption (III) let

$$
\frac{\log (n)}{n^{\theta} h_{n}^{d}}=o(1) \text { for } \theta=\frac{\bar{\alpha}-1-d-\frac{1+\bar{\alpha}}{b-1}}{\bar{\alpha}+3-d-\frac{1+\bar{\alpha}}{b-1}} .
$$

For $\delta_{n}, p_{n}, q_{n}$ from assumption (VI) let

$$
\left(\sqrt{\frac{\log (n)}{n h_{n}^{d}}}+h_{n} p_{n}\right) p_{n} q_{n} \delta_{n}=o\left(n^{-\zeta}\right)
$$

for some $\zeta>0$.

(IX.1) For all $1 \leq t \leq n, n \in \mathbb{N}$ let $f_{t}(\cdot)=f(\cdot)$, for some density $f$.

(IX.2) For all $1 \leq t \leq n, n \in \mathbb{N}$ let $f_{t}(\cdot)=f_{(1)}(\cdot)$ for all $t=1, \ldots,\left\lfloor n s_{0}\right\rfloor$ and $f_{t}(\cdot)=f_{(2)}(\cdot)$ for all $t=\left\lfloor n s_{0}\right\rfloor+1, \ldots, n$, for some densities $f_{(1)}, f_{(2)}$.

Remark The assumptions on the error terms and the mixing assumptions particularly allow for conditional heteroscedasticity. Assumptions (I), (II) and (III) are a trade off 
between the existence of moments and the rate of decay of the mixing coefficient. Assumptions (III), (IV), (VII) and the first part of (VIII) are reproduced from Kristensen (2012). Together with (V) and (VI), they are used to obtain uniform rates of convergence for $\hat{m}_{n}$ stated in Lemma A.1 in the Appendix. In (IX.1), we assume stationarity of the covariates for the whole observation period, while in the case of (IX.2) we assume stationarity before and right after the change occurs. Nevertheless both assumptions rule out general autoregressive effects such as $\boldsymbol{X}_{t}=\left(Y_{t-1}, \ldots, Y_{t-d}\right)$. We will address this issue separately in Sect. 4.

Theorem 3.1 Assume (I), (II), (III), (IV), (V), (VI), (VII) and (VIII). Furthermore let either (IX.1) or (IX.2) hold. Then the change point estimator $\hat{s}_{n}$ is consistent, i.e.

$$
\left|\hat{s}_{n}-s_{0}\right|=o_{P}(1)
$$

Theorem 3.2 Under the assumptions of Theorem 3.1 for the change point estimator $\hat{s}_{n}$ it holds that

$$
\left|\hat{s}_{n}-s_{0}\right|=O_{P}\left(r_{n}^{-1}\right),
$$

where $r_{n}=n$.

The proofs of the theorems can be found in Appendix A.2. We state both theorems seperately since we need Theorem 3.1 to prove Theorem 3.2.

Remark To obtain the rates of convergence we make use of the fact that $\hat{s}_{n}$ can be expressed using the sup norm on $l^{\infty}\left(\mathbb{R}^{d}\right)$, i.e.

$$
N: l^{\infty}\left(\mathbb{R}^{d}\right) \rightarrow \mathbb{R}, g \mapsto N(g):=\sup _{z \in \mathbb{R}^{d}}|g(z)|,
$$

where $l^{\infty}\left(\mathbb{R}^{d}\right)$ is the space of all uniformly bounded real valued functions on $\mathbb{R}^{d}$. Note that similarly $\tilde{\boldsymbol{s}}_{n}$ can be expressed using the $L_{2}(P)$ norm, when $\left(\boldsymbol{X}_{t}\right)_{t}$ is strictly stationary with marginal distribution $P$, namely

$$
\tilde{N}: l^{\infty}\left(\mathbb{R}^{d}\right) \rightarrow \mathbb{R}, g \mapsto \tilde{N}(g):=\left(\int_{\mathbb{R}^{d}}|g(z)|^{2} f(z) d z\right)^{1 / 2}
$$

Using $\tilde{N}(g) \leq N(g)$ for all $g \in l^{\infty}\left(\mathbb{R}^{d}\right)$, corresponding results for $\tilde{s}_{n}$ as in Theorem 3.1 and Theorem 3.2 can be proven in a similar matter.

\section{The autoregressive case}

In this section we will consider the case where the exogenous variables include finitely many lagged values of the endogenous variable, we will refer to this model as the autoregressive case. We will focus on one dimensional covariates, however, the results 
do not depend on the dimension and can also be formulated for higher order autoregression models. Consider the nonparametric autoregression

$$
Y_{t}=m_{t}\left(Y_{t-1}\right)+U_{t}, t=1, \ldots, n,
$$

with unobservable innovations $U_{t}$ and one change in the regression function occurring at some unknown time $\left\lfloor n s_{0}\right\rfloor$ as in (2.2).

Furthermore assume the following.

(IX.3) For all $1 \leq t \leq n, n \in \mathbb{N}$ let $X_{t}:=Y_{t-1}$ be absolutely continuous with density $f_{t}$. Let there exist densities $f_{(1)}$ and $f_{(2)}$ such that $f_{t}(\cdot)=f_{(1)}(\cdot)$ for all $t=1, \ldots,\left\lfloor n s_{0}\right\rfloor$ and $R_{n}(x):=\frac{1}{n} \sum_{j=\left\lfloor n s_{0}\right\rfloor+1}^{n} f_{j}(x)-\frac{n-\left\lfloor n s_{0}\right\rfloor}{n} f_{(2)}(x) \rightarrow 0$ for all $x \in \mathbb{R}$ and $n \rightarrow \infty$.

Remark Note that (IX.3) requires on the one hand strict stationarity up to the time of change $\left\lfloor n s_{0}\right\rfloor$. On the other hand the time series needs to reach its (new) stationary distribution fast enough after the change. This is a generalization of (IX.2) where we assumed stationarity both before and right after the change point, which can not be fulfilled in the model (4.1). A necessary condition then is that there exists a stationary solution of equation (4.1) under both $m_{(1)}(\cdot)$ and $m_{(2)}(\cdot)$ as regression functions.

Example Consider the AR(1)-model

$$
Y_{t}=a_{t} \cdot Y_{t-1}+\varepsilon_{t}
$$

with standard normally distributed innovations $\left(\varepsilon_{t}\right)_{t}$ and $a_{t}=a \in(-1,1)$ for $t \leq$ $\left\lfloor n s_{0}\right\rfloor, a_{t}=b \in(-1,1)$ for $t>\left\lfloor n s_{0}\right\rfloor, a \neq b$. Then assumption (IX.3) is fulfilled. Note to this end that $X_{t}:=Y_{t-1} \sim \mathcal{N}\left(0,1 /\left(1-a^{2}\right)\right)$ for $t \leq\left\lfloor n s_{0}\right\rfloor$. The distribution after the change point is given by $X_{\left\lfloor n s_{0}\right\rfloor+1+k} \sim \mathcal{N}\left(0, b^{2(k+1)} /\left(1-a^{2}\right)+\sum_{i=0}^{k} b^{2 i}\right)$ for all $k>0$. Thus with $\sigma_{j}^{2}:=b^{2\left(j-\left\lfloor n s_{0}\right\rfloor\right)} /\left(1-a^{2}\right)+\sum_{i=0}^{j-\left\lfloor n s_{0}\right\rfloor-1} b^{2 i}$ by the mean value theorem it holds for some $\xi_{j}$ between $\sigma_{j}^{2}$ and $\left(1-b^{2}\right)^{-1}$ that

$$
\begin{aligned}
R_{n}(x) & =\frac{1}{n} \sum_{j=\left\lfloor n s_{0}\right\rfloor+1}^{n}\left(\frac{1}{\sqrt{2 \pi \sigma_{j}^{2}}} \exp \left(-\frac{x^{2}}{2 \sigma_{j}^{2}}\right)-\frac{1}{\sqrt{2 \pi\left(1-b^{2}\right)^{-1}}} \exp \left(-\frac{x^{2}}{2\left(1-b^{2}\right)^{-1}}\right)\right) \\
& =\frac{1}{n} \sum_{j=\left\lfloor n s_{0}\right\rfloor+1}^{n}\left(\sigma_{j}^{2}-\frac{1}{1-b^{2}}\right) \exp \left(-\frac{x^{2}}{2 \xi_{j}}\right)\left(-\frac{1}{2} \cdot \frac{1}{\sqrt{2 \pi \xi_{j}^{\frac{3}{2}}}}+\frac{1}{\left(2 \pi \xi_{j}\right)^{\frac{1}{2}}} \cdot \frac{x^{2}}{2 \xi_{j}^{2}}\right) \\
& \leq C \frac{1}{n} \sum_{j=\left\lfloor n s_{0}\right\rfloor+1}^{n}\left|\sigma_{j}^{2}-\frac{1}{1-b^{2}}\right|
\end{aligned}
$$


for some constant $C<\infty$ for all $x \in \mathbb{R}$. Further we can conclude

$$
\begin{aligned}
\frac{1}{n} \sum_{j=\left\lfloor n s_{0}\right\rfloor+1}^{n}\left|\sigma_{j}^{2}-\frac{1}{1-b^{2}}\right| & =\frac{1}{n} \sum_{j=\left\lfloor n s_{0}\right\rfloor+1}^{n}\left|\frac{b^{2\left(j-\left\lfloor n s_{0}\right\rfloor\right)}}{1-a^{2}}+\frac{1-b^{2\left(j-\left\lfloor n s_{0}\right\rfloor\right)}}{1-b^{2}}-\frac{1}{1-b^{2}}\right| \\
& =\left|\frac{1}{1-a^{2}}-\frac{1}{1-b^{2}}\right| \frac{1}{n} \sum_{j=\left\lfloor n s_{0}\right\rfloor+1}^{n} b^{2\left(j-\left\lfloor n s_{0}\right\rfloor\right)}
\end{aligned}
$$

and thus $R_{n}(x) \underset{n \rightarrow \infty}{\longrightarrow} 0$ for all $x \in \mathbb{R}$.

In general verifying assumption (IX.3) for model (4.1) means to compare the distribution of a stochastic process that is not yet in balance with its stationary distribution. A well known technique to deal with this task is coupling, see e. g. Franke et al. (2002).

Under (IX.3) we get the following consistency result for our change point estimator in the autoregressive case.

Theorem 4.1 Assume model (4.1) under (I), (II), (III), (IV), (V), (VI), (VII), (VIII) and (IX.3). Then the change point estimator $\hat{s}_{n}$ is consistent, i.e.

$$
\left|\hat{s}_{n}-s_{0}\right|=o_{P}(1)
$$

The proof can be found in Appendix A.2.

Remark Another possibility to handle the autoregressive case would be to model the change in a different way, namely

$$
Y_{t}=\left\{\begin{array}{ll}
Y_{t}^{(1)}=m_{(1)}\left(Y_{t-1}^{(1)}\right)+U_{t}^{(1)}, & t=1, \ldots,\left\lfloor n s_{0}\right\rfloor \\
Y_{t}^{(2)}=m_{(2)}\left(Y_{t-1}^{(2)}\right)+U_{t}^{(2)}, & t=\left\lfloor n s_{0}\right\rfloor+1, \ldots, n
\end{array}, \quad m_{(1)} \not \equiv m_{(2)},\right.
$$

for two stationary processes $\left(Y_{t}^{(1)}\right)_{t},\left(Y_{t}^{(2)}\right)_{t}$, see e.g. Kirch et al. (2015). In this case assumption (IX.2) is fulfilled and thus Theorems 3.1 and 3.2 apply.

\section{Finite sample properties}

\subsection{Simulations}

To investigate the finite sample performance of our estimator, we generate data from two different basic models, namely

(IID) $Y_{t}=m_{t}\left(X_{t}\right)+\sigma\left(X_{t}\right) \varepsilon_{t}$, where the observations $\left(X_{t}\right)_{t}$ are i.i.d., univariate and standard normally distributed, just as the errors $\left(\varepsilon_{t}\right)_{t}$.

(TS) $Y_{t}=m_{t}\left(X_{t}\right)+\sigma\left(X_{t}\right) \varepsilon_{t}$, where $\left(\varepsilon_{t}\right)_{t}$ i.i.d. $\sim N(0,1)$ and the univariate observations $\left(X_{t}\right)_{t}$ stem from a time series $X_{t}=0.4 X_{t-1}+\eta_{t}$ with standard normal innovations $\left(\eta_{t}\right)_{t}$. 
(IID)
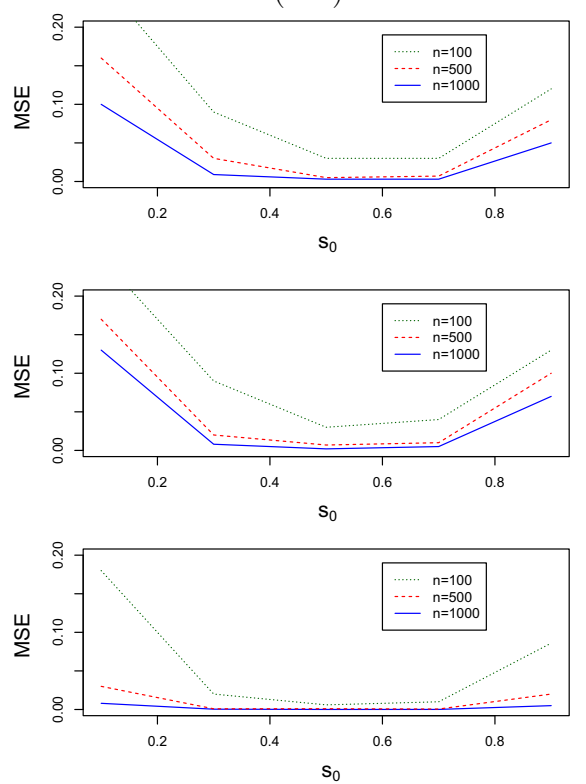

(TS)
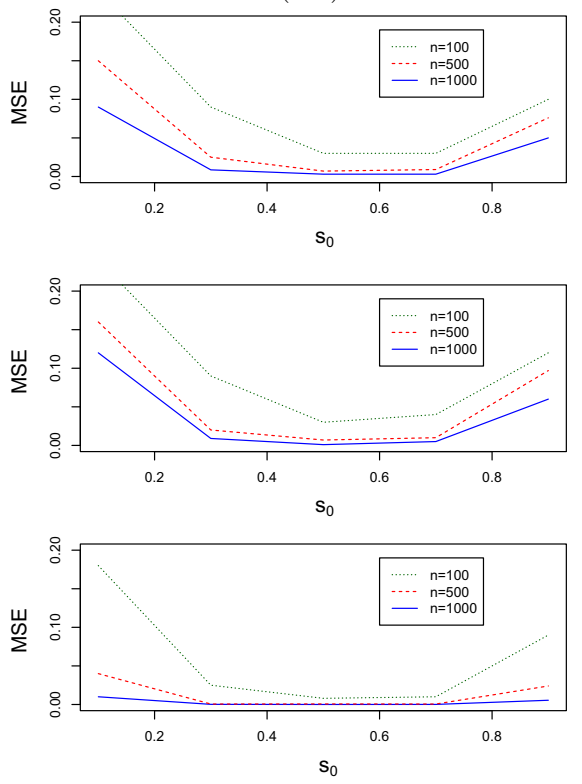

Fig. 1 Simulation results for model (IID) (left), model (TS) (right) and change point scenario (C1) (top), change point scenario (C2) (middle), change point scenario (C3) (bottom)

For both models we generate data both for the homoscedastic case $\sigma \equiv 1$ as well as for the heteroscedastic case $\sigma(x)=\sqrt{1+0.5 x^{2}}$. The results for both are very similar in all situations, thus we only present the results for the heteroscedastic case. To model the change in the regression function we use three different scenarios

(C1) $m_{t}= \begin{cases}-0.5 x, & t=1, \ldots,\left\lfloor n s_{0}\right\rfloor \\ 0.5 x & t=\left\lfloor n s_{0}\right\rfloor+1, \ldots, n,\end{cases}$

(C2) $m_{t}= \begin{cases}0.1 x, & t=1, \ldots,\left\lfloor n s_{0}\right\rfloor \\ 0.9 x & t=\left\lfloor n s_{0}\right\rfloor+1, \ldots, n,\end{cases}$

(C3) $m_{t}= \begin{cases}0.5 x, & t=1, \ldots,\left\lfloor n s_{0}\right\rfloor \\ \left(0.5+3 \exp \left(-0.8 x^{2}\right)\right) x & t=\left\lfloor n s_{0}\right\rfloor+1, \ldots, n,\end{cases}$

where we let $s_{0}$ range from 0.1 to 0.9 . In Fig. 1 the results for 1000 replications and sample sizes $n=100,500,1000$ are shown, where we plot $s_{0}$ against the estimated mean squared error of our estimator $\hat{s}_{n}$. The kernel for $\hat{m}_{n}$ is chosen as the Epanechnikov kernel of order four and the bandwidth is determined by a cross-validation method. It can be seen that our estimator performs quite well even for the smallest sample size $n=100$ when $s_{0}$ is 0.5 or close to it whereas for a change point that lies closer to the boundaries of the observation interval a larger sample size is needed to get satisfying results. This is due to the fact that if $s_{0}=0.1$ or $s_{0}=0.9$ there are only 10 observations before and after the change point respectively for $n=100$ and thus the estimation of $m_{(1)}$ and $m_{(2)}$ respectively are poor. Moreover an asymmetry 
(IID)

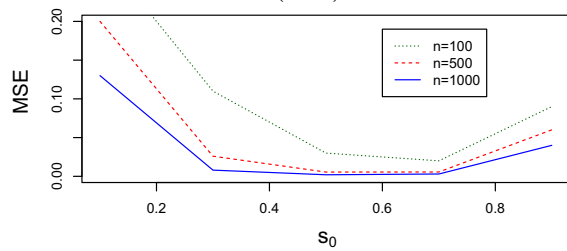

(TS)

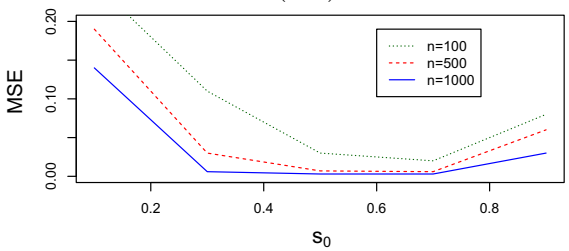

Fig. 2 Simulation results for model (IID) (left) and model (TS) (right) with change point scenario (C1) and an additional change in the variance function
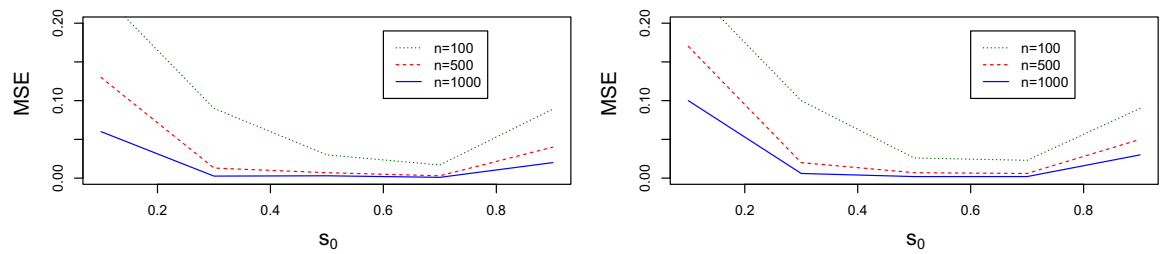

Fig. 3 Simulation results for model (AR) and change point scenario (C1) (left), change point scenario (C2) (right)

in the results is striking. This stems from the CUSUM type statistic that our estimator is based on. For $s_{0}=0.1 \mathrm{e}$. g. the sum consists of only $0.1 n$ summands and thus the estimation of e.g. $E\left[U_{t}\right]$ is worse than if $s_{0}=0.9$ and the estimation is based on $0.9 n$ summands. The effect of a decreasing performance of the estimators the closer $s_{0}$ gets to the boundaries is typical for change point estimators based on CUSUM statistics and can be antagonized by the use of appropriate weights, see e. g. Ferger (2005).

To stress our estimator a little further we simulate the scenario that there is also a change in the variance function $\sigma$ at a different time point than the change in the regression function $m$. In this situation the estimator should still be able to detect $s_{0}$, the change point in the regression function. The results are shown in Fig. 2 for model (IID) and model (TS) with change point scenario (C1) where $\sigma_{t}(x)=\sqrt{1+0.1 x^{2}}$ for $t \leq 0.4 n$ and $\sigma_{t}(x)=\sqrt{1+0.8 x^{2}}$ for $t>0.4 n$. They confirm the good performance of our estimator even in this more difficult situation.

As discussed in Sect. 4 our estimator can also be applied to the autoregressive case. To investigate the finite sample performance in this situation we generate data according to the model

(AR) $Y_{t}=m_{t}\left(Y_{t-1}\right)+\sigma\left(Y_{t-1}\right) \varepsilon_{t}$, where $\left(\varepsilon_{t}\right)_{t}$ i.i.d. $\sim N(0,1)$.

For $\sigma \equiv 1$ and change point scenario (C1) as well as (C2) assumption (IX.3) is fulfilled, see the example in Sect. 4. Simulation results for these cases are shown in Fig. 3 where the setting is the same as described above. They look very similar to the results of model (IID) and (TS) and thus confirm the theoretical result of Theorem 4.1. Even for examples where assumption (IX.3) can not be verified easily the performance of our estimator is satisfying, see Fig. 4 for model (AR) with $\sigma \equiv 1$ and change point scenario (C3) as well as the heteroscedastic model (AR) with $\sigma=\sqrt{1+0.5 x^{2}}$ and change point scenario $(\mathrm{C} 1)$. 

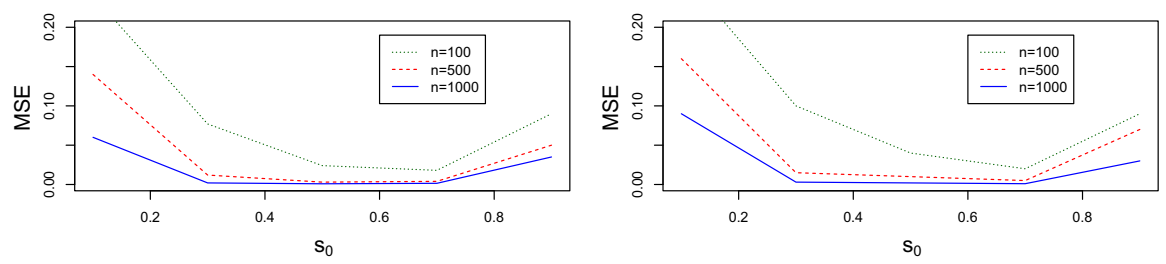

Fig. 4 Simulation results for homoscedastic model (AR) with change point scenario (C3) (left) and heteroscedastic model (AR) with change point scenario (C1) (right)

As stated in the remark in Sect. 2 it is also possible to base the estimator on a Cramér-von Mises type functional of the marked empirical process of residuals. The simulation results for this type of estimator are very similar to those presented here for the Kolmogorov-Smirnov type estimator $\hat{s}_{n}$ and are omitted for the sake of brevity.

\subsection{Data example}

Finally, we will consider a real data example. The data at hand contains 36 measurements of the annual flow volume of the small Czech river, Ráztoka, recorded between 1954 and 1989 as well as the annual rainfall during that time. It was considered by Hušková and Antoch (2003) to investigate the effect of controlled deforestation on the capability for water retention of the soil. To this end it is of interest if and when the relationship between rainfall and flow volume changes. We set $X_{t}$ as the annual rainfall and $Y_{t}$ as the annual flow volume. Mohr and Neumeyer (2019) applied their Kolmogorov-Smirnov test to this data set, which clearly rejects the null of no change in the conditional mean function, indicating the existence of a change in the relationship between rainfall and flow volume. Using $\hat{s}_{n}$ to estimate the unknown time of change suggests a change in 1979. Note that this is consistent with the literature. As was pointed out by Hušková and Antoch (2003) large scale deforestation had started around that time. Figure 5 shows on the left-hand side the scatterplot $X_{t}$ against $Y_{t}$ using dots for the observations after the estimated change and crosses for the observations before the estimated change. On the right-hand side the figure shows the cumulative sum, $n^{1 / 2} \sup _{z \in \mathbb{R}}\left|\hat{T}_{n}(\cdot, z)\right|$, as well as the critical value of the test used in Mohr and Neumeyer (2019) (red horizontal line) and the estimated change (green vertical line). Note that $\tilde{s}_{n}$ leads to the same result.

\section{Concluding remarks}

In this paper we consider nonparametric regression models with a change in the unknown regression function that allows for time series data as well as conditional heteroscedasticity. We propose an estimator for the rescaled change point that is based on the sequential marked empirical process of residuals and show consistency as well as a rate of convergence of $O_{P}\left(n^{-1}\right)$. In an autoregressive setting we additionally give a consistency result for the proposed estimator. 

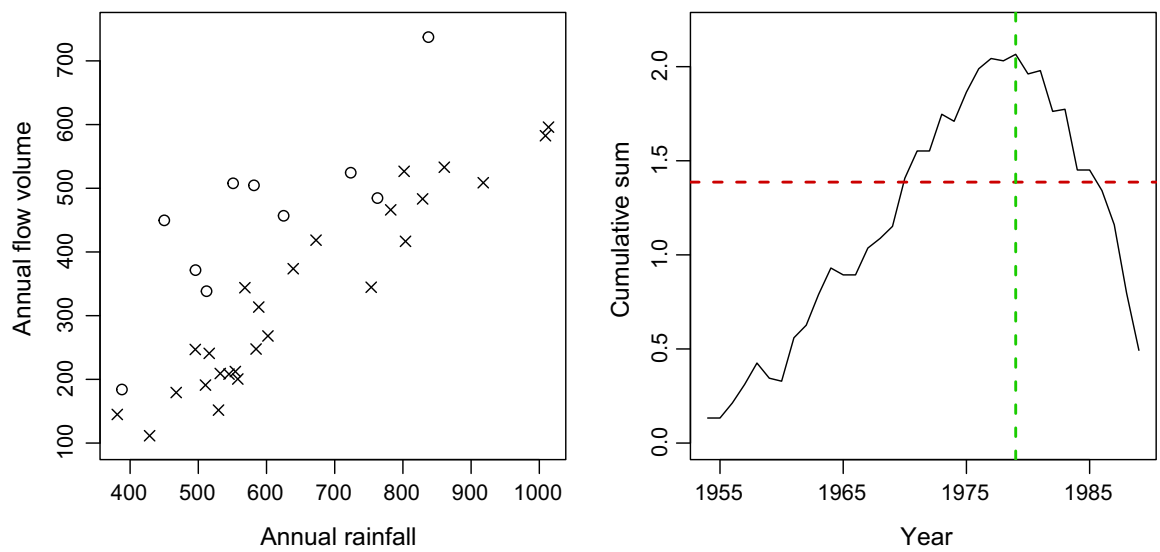

Fig. 5 Ráztoka data: scatterplot (left) and CUSUM (right)

If more than one change occurs, the proposed estimator is not consistent for one of the changes in some situations. For detecting multiple changes we refer the reader to alternative procedures such as the MOSUM procedure proposed by Eichinger and Kirch (2018) or the wild binary segmentation procedure by Fryzlewicz (2014) (see also Fryzlewicz (2019)).

Investigating the asymptotic distribution of the proposed estimator is a subsequent issue. Certainly, it is of great interest as it can be used to obtain confidence intervals. However, this subject goes beyond the scope of the paper at hand and is postponed to future research.

Acknowledgements Open Access funding provided by Projekt DEAL. Financial support by the DFG (Research Unit FOR 1735 Structural Inference in Statistics: Adaptation and Efficiency) is gratefully acknowledged.

Open Access This article is licensed under a Creative Commons Attribution 4.0 International License, which permits use, sharing, adaptation, distribution and reproduction in any medium or format, as long as you give appropriate credit to the original author(s) and the source, provide a link to the Creative Commons licence, and indicate if changes were made. The images or other third party material in this article are included in the article's Creative Commons licence, unless indicated otherwise in a credit line to the material. If material is not included in the article's Creative Commons licence and your intended use is not permitted by statutory regulation or exceeds the permitted use, you will need to obtain permission directly from the copyright holder. To view a copy of this licence, visit http://creativecommons.org/licenses/by/4.0/.

\section{Appendix: Proofs}

\section{A.1 Auxiliary results}

Lemma A.1 Under the assumptions (III), (IV), (V), (VI), (VII) and (VIII), it holds that

$$
\sup _{\boldsymbol{x} \in \boldsymbol{J}_{n}}\left|\hat{m}_{n}(\boldsymbol{x})-\bar{m}_{n}(\boldsymbol{x})\right|=O_{P}\left(\left(\sqrt{\frac{\log (n)}{n h_{n}^{d}}}+h_{n} p_{n}\right) \delta_{n} p_{n} q_{n}\right)
$$


where

$$
\bar{m}_{n}(\boldsymbol{x})=\frac{\sum_{i=1}^{n} f_{i}(\boldsymbol{x}) m_{i}(\boldsymbol{x})}{\sum_{i=1}^{n} f_{i}(\boldsymbol{x})}
$$

The proof is similar to the proof of Lemma 2.2 in Mohr (2018). The key tool is an application of Theorem 1 in Kristensen (2009). Details are omitted for the sake of brevity.

Remark Under (IX.1) we have

$$
\bar{m}_{n}(\boldsymbol{x})=\frac{\left\lfloor n s_{0}\right\rfloor}{n} m_{(1)}(\boldsymbol{x})+\frac{n-\left\lfloor n s_{0}\right\rfloor}{n} m_{(2)}(\boldsymbol{x}),
$$

under (IX.2) and (IX.3) we have

$$
\bar{m}_{n}(\boldsymbol{x})=\frac{\frac{\left\lfloor n s_{0}\right\rfloor}{n} f_{(1)}(\boldsymbol{x})}{\bar{f}_{n}(\boldsymbol{x})}\left(m_{(1)}(\boldsymbol{x})-m_{(2)}(\boldsymbol{x})\right)+m_{(2)}(\boldsymbol{x}),
$$

where

$$
\bar{f}_{n}(\boldsymbol{x}):=\frac{1}{n} \sum_{i=1}^{n} f_{i}(\boldsymbol{x})= \begin{cases}\frac{\left\lfloor n s_{0}\right\rfloor}{n} f_{(1)}(\boldsymbol{x})+\frac{n-\left\lfloor n s_{0}\right\rfloor}{n} f_{(2)}(\boldsymbol{x}), & \text { for (IX.2) } \\ \frac{\left\lfloor n s_{0}\right\rfloor}{n} f_{(1)}(\boldsymbol{x})+\frac{n-\left\lfloor n s_{0}\right\rfloor}{n} f_{(2)}(\boldsymbol{x})+R_{n}(\boldsymbol{x}), & \text { for (IX.3) }\end{cases}
$$

with $R_{n}(\cdot)$ from assumption (IX.3).

Lemma A.2 Under the assumptions of Theorem 3.1 as well as under those of Theorem 4.1 there exists a constant $\bar{C}=\bar{C}(C)<\infty$ such that

$$
P\left(\sup _{s \in[0,1]} \sup _{z \in \mathbb{R}^{d}}\left|\sum_{i=L+1}^{L+\left\lfloor\kappa_{n} s\right\rfloor} U_{i} \omega_{n}\left(\boldsymbol{X}_{i}\right) I\left\{\boldsymbol{X}_{i} \leq \boldsymbol{z}\right\}\right|>C \kappa_{n}\right) \leq \bar{C} \kappa_{n}^{\frac{1}{q}-1}
$$

for all $L=0,1, \ldots, n-\kappa_{n}, 1 \leq \kappa_{n} \leq n, n \in \mathbb{N}$ and all $C>0$ with q from assumption (I).

Proof of Lemma A.2 The proof follows along similar lines as the proof of Lemma A.3 in Mohr (2018). Throughout the proof the values of $C$ and $\bar{C}$ may vary from line to line but they are always positive, finite and independent of $n$. Further note that deterministic terms that are of order $O\left(\kappa_{n}\right)$ can be omitted as we can choose constants appropriately. It holds that

$$
\begin{aligned}
& \sup _{s \in[0,1]} \sup _{z \in \mathbb{R}^{d}}\left|\sum_{i=L+1}^{L+\left\lfloor\kappa_{n} s\right\rfloor} U_{i} \omega_{n}\left(\boldsymbol{X}_{i}\right) I\left\{\boldsymbol{X}_{i} \leq \boldsymbol{z}\right\}\right| \\
& =\sup _{s \in[0,1]} \sup _{z \in \mathbb{R}^{d}}\left|\sum_{i=L+1}^{L+\left\lfloor\kappa_{n} s\right\rfloor} U_{i} \omega_{n}\left(\boldsymbol{X}_{i}\right) I\left\{\boldsymbol{X}_{i} \leq \boldsymbol{z}\right\}-E\left[\sum_{i=L+1}^{L+\left\lfloor\kappa_{n} s\right\rfloor} U_{i} \omega_{n}\left(\boldsymbol{X}_{i}\right) I\left\{\boldsymbol{X}_{i} \leq \boldsymbol{z}\right\}\right]\right|
\end{aligned}
$$




$$
\begin{array}{r}
\leq \sup _{s \in[0,1]} \sup _{z \in \mathbb{R}^{d}}\left|\sum_{i=L+1}^{L+\left\lfloor\kappa_{n} s\right\rfloor} U_{i} I\left\{\left|U_{i}\right|>\kappa_{n}^{\frac{1}{q}}\right\} \omega_{n}\left(\boldsymbol{X}_{i}\right) I\left\{\boldsymbol{X}_{i} \leq z\right\}-E\left[\sum_{i=L+1}^{L+\left\lfloor\kappa_{n} s\right\rfloor} U_{i} I\left\{\left|U_{i}\right|>\kappa_{n}^{\frac{1}{q}}\right\} \omega_{n}\left(\boldsymbol{X}_{i}\right) I\left\{\boldsymbol{X}_{i} \leq \boldsymbol{z}\right\}\right]\right| \\
+\sup _{s \in[0,1]} \sup _{z \in \mathbb{R}^{d}}\left|\sum_{i=L+1}^{L+\left\lfloor\kappa_{n} s\right\rfloor} U_{i} I\left\{\left|U_{i}\right| \leq \kappa_{n}^{\frac{1}{q}}\right\} \omega_{n}\left(\boldsymbol{X}_{i}\right) I\left\{\boldsymbol{X}_{i} \leq z\right\}-E\left[\sum_{i=L+1}^{L+\left\lfloor\kappa_{n} s\right\rfloor} U_{i} I\left\{\left|U_{i}\right| \leq \kappa_{n}^{\frac{1}{q}}\right\} \omega_{n}\left(\boldsymbol{X}_{i}\right) I\left\{\boldsymbol{X}_{i} \leq \boldsymbol{z}\right\}\right]\right|
\end{array}
$$

where (A.1) is of the desired rate in probability since

$$
P\left(\sum_{i=L+1}^{L+\kappa_{n}}\left|U_{i}\right| I\left\{\left|U_{i}\right|>\kappa_{n}^{\frac{1}{q}}\right\}>C \kappa_{n}\right) \leq C^{-1} C_{U} \kappa_{n}^{\frac{1}{q}-1}
$$

by Markov's inequality with

$$
\begin{aligned}
E\left[\left|U_{i}\right| I\left\{\left|U_{i}\right|>\kappa_{n}^{\frac{1}{q}}\right\}\right] & =E\left[\left|U_{i}\right|^{q}\left|U_{i}\right|^{-(q-1)} I\left\{\left|U_{i}\right|>\kappa_{n}^{\frac{1}{q}}\right\}\right] \\
& \leq \kappa_{n}^{-\frac{q-1}{q}} E\left[\left|U_{i}\right|^{q}\right] \\
& \leq C_{U} \kappa_{n}^{\frac{1}{q}-1} \quad \text { for all } i \text { and for } C_{U}<\infty \text { from assumption (I). }
\end{aligned}
$$

Considering the term (A.2) we define the function class

$$
\mathcal{F}_{n}:=\left\{(u, \boldsymbol{x}) \mapsto u I\left\{|u| \leq \kappa_{n}^{\frac{1}{q}}\right\} \omega_{n}(\boldsymbol{x}) I\{\boldsymbol{x} \leq z\}: z \in \mathbb{R}^{d}\right\}
$$

to rewrite the assertion as

$$
P\left(\sup _{s \in[0,1]} \sup _{\varphi \in \mathcal{F}_{n}}\left|\sum_{i=L+1}^{L+\left\lfloor\kappa_{n} s\right\rfloor}\left(\varphi\left(U_{i}, \boldsymbol{X}_{i}\right)-\int \varphi d P\right)\right|>C \kappa_{n}\right) \leq \bar{C} \kappa_{n}^{\frac{1}{q}-1}
$$

Now we will cover $[0,1]$ by finitely many intervals and $\mathcal{F}_{n}$ by finitely many brackets to replace the supremum by a maximum. Let therefore

$$
0=s_{1}<\cdots<s_{K_{n}}=1
$$


part the interval $[0,1]$ in $K_{n}$ subintervals of length $\bar{\epsilon}_{n}$ with $\bar{\epsilon}_{n}=\kappa_{n}^{-\frac{1}{q}}$. Then

$$
\begin{aligned}
& \sup _{s \in[0,1]} \sup _{\varphi \in \mathcal{F}_{n}}\left|\sum_{i=L+1}^{L+\left\lfloor\kappa_{n} s\right\rfloor}\left(\varphi\left(U_{i}, \boldsymbol{X}_{i}\right)-\int \varphi d P\right)\right| \\
& =\max _{k} \sup _{\substack{s \in[0,1] \\
\left|s-s_{k}\right| \leq \bar{\epsilon}_{n}}} \sup _{\varphi \in \mathcal{F}_{n}}\left|\sum_{i=L+1}^{L+\left\lfloor\kappa_{n} s\right\rfloor}\left(\varphi\left(U_{i}, \boldsymbol{X}_{i}\right)-\int \varphi d P\right)\right| \\
& \leq \max _{k} \sup _{\varphi \in \mathcal{F}_{n}}\left|\sum_{i=L+1}^{L+\left\lfloor\kappa_{n} s_{k}\right\rfloor}\left(\varphi\left(U_{i}, \boldsymbol{X}_{i}\right)-\int \varphi d P\right)\right| \\
& +\max _{k} \sup _{\substack{s \in[0,1] \\
\left|s-s_{k}\right| \leq \bar{\epsilon}_{n}}} \sup _{\varphi \in \mathcal{F}_{n}} \sum_{i=L+1}^{L+\kappa_{n}} \underbrace{\left|\varphi\left(U_{i}, \boldsymbol{X}_{i}\right)-\int \varphi d P\right|}_{\leq 2 \kappa_{n}^{\frac{1}{q}}} \mid I\left\{\frac{i-L}{\kappa_{n}} \leq s\right\} \\
& -I\left\{\frac{i-L}{\kappa_{n}} \leq s_{k}\right\} \mid \\
& \leq \max _{k} \sup _{\varphi \in \mathcal{F}_{n}}\left|\sum_{i=L+1}^{L+\left\lfloor\kappa_{n} s_{k}\right\rfloor}\left(\varphi\left(U_{i}, \boldsymbol{X}_{i}\right)-\int \varphi d P\right)\right|+2 \kappa_{n}^{\frac{1}{q}}\left(\kappa_{n} \bar{\epsilon}_{n}+1\right)
\end{aligned}
$$

and $2 \kappa_{n}^{\frac{1}{q}}\left(\kappa_{n} \bar{\epsilon}_{n}+1\right)=2\left(\kappa_{n}+\kappa_{n}^{\frac{1}{q}}\right)=O\left(\kappa_{n}\right)$. Further let

$$
\begin{aligned}
\varphi_{j}^{u}(u, \boldsymbol{x}):= & u I\left\{|u| \leq \kappa_{n}^{\frac{1}{q}}\right\} I\{u \geq 0\} \omega_{n}(\boldsymbol{x}) I\left\{\boldsymbol{x} \leq \boldsymbol{z}_{\boldsymbol{j}}\right\} \\
& +u I\left\{|u| \leq \kappa_{n}^{\frac{1}{q}}\right\} I\{u<0\} \omega_{n}(\boldsymbol{x}) I\left\{\boldsymbol{x} \leq \boldsymbol{z}_{j-1}\right\}
\end{aligned}
$$

and

$$
\begin{aligned}
\varphi_{\boldsymbol{j}}^{l}(u, \boldsymbol{x}):= & u I\left\{|u| \leq \kappa_{n}^{\frac{1}{q}}\right\} I\{u \geq 0\} \omega_{n}(\boldsymbol{x}) I\left\{\boldsymbol{x} \leq \boldsymbol{z}_{\boldsymbol{j}-\mathbf{1}}\right\} \\
& +u I\left\{|u| \leq \kappa_{n}^{\frac{1}{q}}\right\} I\{u<0\} \omega_{n}(\boldsymbol{x}) I\left\{\boldsymbol{x} \leq \boldsymbol{z}_{\boldsymbol{j}}\right\}
\end{aligned}
$$

form the brackets $\left[\varphi_{j}^{l}, \varphi_{j}^{u}\right]_{j \in \times_{i=1}^{d}\left\{1, \ldots, N_{i}\right\}}$ of $\mathcal{F}_{n}$, where $z_{j}:=\left(z_{j_{1}, 1}, \ldots, z_{j_{d}, d}\right)$ and

$$
-\infty=z_{0, i}<\cdots<z_{N_{i}, i}=\infty
$$

gives a partition of $\mathbb{R}$ for all $i=1, \ldots, d$. Then for all $z \in \mathbb{R}^{d}$ there exists a $j \in$ $\times_{i=1}^{d}\left\{1, \ldots, N_{i}\right\}$ such that $z_{j-1}<z \leq z_{j}$ where $\boldsymbol{j}-\mathbf{1}:=\left(j_{1}-1, \ldots, j_{d}-1\right)$. Thus every element $\varphi$ of $\mathcal{F}_{n}$ lies in one of the brackets $\left[\varphi_{j}^{l}, \varphi_{j}^{u}\right]$, i. e. $\varphi_{j}^{l}(u, \boldsymbol{x}) \leq \varphi(u, \boldsymbol{x}) \leq$ $\varphi_{j}^{u}(u, \boldsymbol{x})$ for all $(u, \boldsymbol{x})$. We say a bracket $\left[\varphi_{j}^{l}, \varphi_{j}^{u}\right]$ is of size $\epsilon_{n}$ if $\int\left(\varphi_{j}^{u}-\varphi_{j}^{l}\right) d P \leq \epsilon_{n}$. The total number of brackets of size $\epsilon_{n}$ needed to cover $\mathcal{F}_{n}$ is denoted by $J_{n}:=$ 
$N_{[]}\left(\epsilon_{n}, \mathcal{F}_{n},\|\cdot\|_{L_{1}(P)}\right)$ and is of order $J_{n}=O\left(\epsilon_{n}^{-d}\right)$, which follows analogously to but easier than the proof of Lemma A.7 in Mohr (2018).

For all $\varphi \in \mathcal{F}_{n}$ there exists a $j$ with $\varphi_{j}^{l} \leq \varphi \leq \varphi_{j}^{u}$ and thus

$$
\varphi-\int \varphi d P \leq \varphi_{j}^{u}-\int \varphi_{j}^{u} d P+\int\left(\varphi_{j}^{u}-\varphi_{j}^{l}\right) d P
$$

and

$$
\varphi-\int \varphi d P \geq \varphi_{j}^{l}-\int \varphi_{j}^{l} d P-\int\left(\varphi_{j}^{u}-\varphi_{j}^{l}\right) d P
$$

Therefore for all $s \in[0,1]$

$$
\begin{aligned}
& \sup _{\varphi \in \mathcal{F}_{n}}\left|\sum_{i=L+1}^{L+\left\lfloor\kappa_{n} s\right\rfloor}\left(\varphi\left(U_{i}, \boldsymbol{X}_{i}\right)-\int \varphi d P\right)\right| \\
& =\max _{j} \sup _{\varphi \in\left[\varphi_{j}^{l}, \varphi_{j}^{u}\right] \mid}\left|\sum_{i=L+1}^{L+\left\lfloor\kappa_{n} s\right\rfloor}\left(\varphi\left(U_{i}, \boldsymbol{X}_{i}\right)-\int \varphi d P\right)\right| \\
& \leq \max _{j} \max _{j}\left\{\left|\sum_{i=L+1}^{L+\left\lfloor\kappa_{n} s\right\rfloor}\left(\varphi_{j}^{u}\left(U_{i}, \boldsymbol{X}_{i}\right)-\int \varphi_{j}^{u} d P\right)\right|, \mid \sum_{i=L+1}^{L+\left\lfloor\kappa_{n} s\right\rfloor}\left(\varphi_{j}^{l}\left(U_{i}, \boldsymbol{X}_{i}\right)\right.\right. \\
& \left.\left.\quad-\int \varphi_{j}^{l} d P\right) \mid\right\} \\
& \quad+\kappa_{n} \max _{j} \underbrace{\left.\int \varphi_{j}^{u}-\varphi_{j}^{l}\right) d P}_{\leq \epsilon_{n}}
\end{aligned}
$$

and $\kappa_{n} \epsilon_{n}=O\left(\kappa_{n}\right)$ if we choose $\epsilon_{n}$ constant. Thus it remains to show that

$$
P\left(\max _{\boldsymbol{j}, k}\left|\sum_{i=L+1}^{L+\left\lfloor\kappa_{n} s_{k}\right\rfloor}\left(\varphi_{\boldsymbol{j}}^{u}\left(U_{i}, \boldsymbol{X}_{i}\right)-\int \varphi_{j}^{u} d P\right)\right|>C \kappa_{n}\right) \leq \bar{C} \kappa_{n}^{\frac{1}{q}-1}
$$

and the same with $\varphi_{j}^{u}$ replaced by $\varphi_{j}^{l}$. Recall that

$$
\begin{aligned}
& \max _{\boldsymbol{j}, k}\left|\sum_{i=L+1}^{L+\left\lfloor\kappa_{n} s_{k}\right\rfloor}\left(\varphi_{\boldsymbol{j}}^{u}\left(U_{i}, \boldsymbol{X}_{i}\right)-\int \varphi_{j}^{u} d P\right)\right| \\
& \leq \max _{\boldsymbol{j}, k} \mid \sum_{i=L+1}^{L+\left\lfloor\kappa_{n} s_{k}\right\rfloor}\left(U_{i} I\left\{\left|U_{i}\right| \leq \kappa_{n}^{\frac{1}{q}}\right\} I\left\{U_{i} \geq 0\right\} \omega_{n}\left(\boldsymbol{X}_{i}\right) I\left\{\boldsymbol{X}_{i} \leq z_{j}\right\}\right. \\
& \left.\quad-E\left[U_{i} I\left\{\left|U_{i}\right| \leq \kappa_{n}^{\frac{1}{q}}\right\} I\left\{U_{i} \geq 0\right\} \omega_{n}\left(\boldsymbol{X}_{i}\right) I\left\{\boldsymbol{X}_{i} \leq z_{j}\right\}\right]\right) \mid
\end{aligned}
$$




$$
\begin{aligned}
& +\max _{\boldsymbol{j}, k} \mid \sum_{i=L+1}^{L+\left\lfloor\kappa_{n} s_{k}\right\rfloor}\left(U_{i} I\left\{\left|U_{i}\right| \leq \kappa_{n}^{\frac{1}{q}}\right\} I\left\{U_{i}<0\right\} \omega_{n}\left(\boldsymbol{X}_{i}\right) I\left\{\boldsymbol{X}_{i} \leq z_{j-\mathbf{1}}\right\}\right. \\
& \left.-E\left[U_{i} I\left\{\left|U_{i}\right| \leq \kappa_{n}^{\frac{1}{q}}\right\} I\left\{U_{i}<0\right\} \omega_{n}\left(\boldsymbol{X}_{i}\right) I\left\{\boldsymbol{X}_{i} \leq z_{j-1}\right\}\right]\right) \mid
\end{aligned}
$$

We will only consider the first summand in more detail since the rest works analogously. To prove that (A.3) is stochastically of the desired rate we apply a Bernstein type inequality for $\alpha$-mixing processes, see Liebscher (1996) Therorem 2.1. Following his notation we define

$$
\begin{aligned}
Z_{i}:= & \left(U_{i+L} I\left\{\left|U_{i+L}\right| \leq \kappa_{n}^{\frac{1}{q}}\right\} I\left\{U_{i+L} \geq 0\right\} \omega_{n}\left(\boldsymbol{X}_{i+L}\right) I\left\{\boldsymbol{X}_{i+L} \leq z\right\}\right. \\
& \left.-E\left[U_{i+L} I\left\{\left|U_{i+L}\right| \leq \kappa_{n}^{\frac{1}{q}}\right\} I\left\{U_{i+L} \geq 0\right\} \omega_{n}\left(\boldsymbol{X}_{i+L}\right) I\left\{\boldsymbol{X}_{i+L} \leq z\right\}\right]\right) I\left\{\frac{i}{\kappa_{n}} \leq s_{k}\right\}
\end{aligned}
$$

for fixed $z \in \mathbb{R}^{d}$ and $s \in[0,1]$. Note that $\left|Z_{i}\right| \leq 2 \kappa_{n}^{\frac{1}{q}}=: S\left(\kappa_{n}\right), Z_{i}$ is centered and

$$
D\left(\kappa_{n}, N\right):=\sup _{0 \leq T \leq \kappa_{n}-1} E\left[\left(\sum_{j=T+1}^{(T+N) \wedge \kappa_{n}} Z_{j}\right)^{2}\right] \leq N^{2} E\left[Z_{i}^{2}\right] \leq C_{U} N^{2}
$$

by assumption (I). Thus Liebscher's Theorem can be applied with $N=\left\lfloor\kappa_{n}^{1-\frac{2}{q}}\right\rfloor$. This means that

$$
\begin{aligned}
& P\left(\max _{\boldsymbol{j}, k} \mid \sum_{i=L+1}^{L+\left\lfloor\kappa_{n} s_{k}\right\rfloor} U_{i} I\left\{\left|U_{i}\right| \leq \kappa_{n}^{\frac{1}{q}}\right\} I\left\{U_{i} \geq 0\right\} \omega_{n}\left(\boldsymbol{X}_{i}\right) I\left\{\boldsymbol{X}_{i} \leq \boldsymbol{z}_{j}\right\}\right. \\
& \left.-E\left[U_{i} I\left\{\left|U_{i}\right| \leq \kappa_{n}^{\frac{1}{q}}\right\} I\left\{U_{i} \geq 0\right\} \omega_{n}\left(\boldsymbol{X}_{i}\right) I\left\{\boldsymbol{X}_{i} \leq z_{j}\right\}\right] \mid>C \kappa_{n}\right) \\
& \leq \sum_{j, k} P\left(\mid \sum_{i=L+1}^{L+\left\lfloor\kappa_{n} s_{k}\right\rfloor}\left(U_{i} I\left\{\left|U_{i}\right| \leq \kappa_{n}^{\frac{1}{q}}\right\} I\left\{U_{i} \geq 0\right\} \omega_{n}\left(\boldsymbol{X}_{i}\right) I\left\{\boldsymbol{X}_{i} \leq z_{j}\right\}\right.\right. \\
& \left.\left.-E\left[U_{i} I\left\{\left|U_{i}\right| \leq \kappa_{n}^{\frac{1}{q}}\right\} I\left\{U_{i} \geq 0\right\} \omega_{n}\left(\boldsymbol{X}_{i}\right) I\left\{\boldsymbol{X}_{i} \leq z_{j}\right\}\right]\right) \mid>C \kappa_{n}\right) \\
& \leq J_{n} K_{n}\left(4 \exp \left(-\frac{C^{2} \kappa_{n}^{2}}{64 \frac{\kappa_{n}}{N} D\left(\kappa_{n}, N\right)+\frac{8}{3} C \kappa_{n} N S\left(\kappa_{n}\right)}\right)+4 \frac{\kappa_{n}}{N} \alpha(N)\right) \\
& \leq J_{n} K_{n}\left(4 \exp \left(-\frac{C^{2} \kappa_{n}^{2}}{64 C_{U} \kappa_{n}^{2-\frac{2}{q}}+\frac{16}{3} C \kappa_{n}^{2-\frac{1}{q}}}\right)+4 \kappa_{n}^{\frac{2}{q}} \alpha\left(\kappa_{n}^{1-\frac{2}{q}}\right)\right) \\
& \leq J_{n} K_{n}\left(4 \exp \left(-C_{1} \kappa_{n}^{\frac{1}{q}}\right)+4 \kappa_{n}^{\frac{2}{q}} \alpha\left(\kappa_{n}^{1-\frac{2}{q}}\right)\right)
\end{aligned}
$$




$$
\begin{aligned}
& \leq C_{2} \kappa_{n}^{\frac{1}{q}}\left(\left(C_{1} \kappa_{n}^{\frac{1}{q}}\right)^{-q}+\kappa_{n}^{\frac{2}{q}-\bar{\alpha}+\frac{2 \bar{\alpha}}{q}}\right) \\
& \leq \bar{C} \kappa_{n}^{\frac{1}{q}-1}
\end{aligned}
$$

for some constants $C_{1}, C_{2}, \bar{C}$ where the second to last inequality follows from the fact that $\exp (-x)<x^{-k} k$ ! for all $k \in \mathbb{N}$ and $x \in \mathbb{R}_{+}$and the last inequality is true by assumption (III) which implies $\bar{\alpha}>(q+2) /(q-2)$. This completes the proof.

Lemma A.3 Under the assumptions of Theorem 3.1 as well as under those of Theorem 4.1 there exists a constant $\bar{C}=\bar{C}(C)<\infty$ such that

$$
\begin{aligned}
& P\left(\sup _{s \in[0,1]} \sup _{z \in \mathbb{R}^{d}} \mid \sum_{i=L+1}^{\left(L+\left\lfloor\kappa_{n} s\right\rfloor\right) \wedge\left\lfloor n s_{0}\right\rfloor}\left(\left(m_{(1)}\left(\boldsymbol{X}_{i}\right)-\bar{m}_{n}\left(\boldsymbol{X}_{i}\right)\right) \omega_{n}\left(\boldsymbol{X}_{i}\right) I\left\{\boldsymbol{X}_{i} \leq z\right\}\right.\right. \\
& \left.\left.-E\left[\left(m_{(1)}\left(\boldsymbol{X}_{i}\right)-\bar{m}_{n}\left(\boldsymbol{X}_{i}\right)\right) \omega_{n}\left(\boldsymbol{X}_{i}\right) I\left\{\boldsymbol{X}_{i} \leq z\right\}\right]\right) \mid>C \kappa_{n}\right) \leq \bar{C} \kappa_{n}^{\frac{1}{r}-1}
\end{aligned}
$$

and

$$
\begin{aligned}
& P\left(\sup _{s \in[0,1]} \sup _{z \in \mathbb{R}^{d}} \mid \sum_{i=L \vee\left\lfloor n s_{0}\right\rfloor+1}^{L+\left\lfloor\kappa_{n} s\right\rfloor}\left(\left(m_{(2)}\left(\boldsymbol{X}_{i}\right)-\bar{m}_{n}\left(\boldsymbol{X}_{i}\right)\right) \omega_{n}\left(\boldsymbol{X}_{i}\right) I\left\{\boldsymbol{X}_{i} \leq \boldsymbol{z}\right\}\right.\right. \\
& \left.\left.-E\left[\left(m_{(2)}\left(\boldsymbol{X}_{i}\right)-\bar{m}_{n}\left(\boldsymbol{X}_{i}\right)\right) \omega_{n}\left(\boldsymbol{X}_{i}\right) I\left\{\boldsymbol{X}_{i} \leq \boldsymbol{z}\right\}\right]\right) \mid>C \kappa_{n}\right) \leq \bar{C} \kappa_{n}^{\frac{1}{r}-1}
\end{aligned}
$$

for all $L=0,1, \ldots, n-\kappa_{n}, 1 \leq \kappa_{n} \leq n, n \in \mathbb{N}$ and all $C>0$ with $r$ from assumption (II).

Proof of Lemma A.3 First we will distinguish between the cases $L+\left\lfloor\kappa_{n} s\right\rfloor \leq\left\lfloor n s_{0}\right\rfloor$ and $L+\left\lfloor\kappa_{n} s\right\rfloor>\left\lfloor n s_{0}\right\rfloor$. In the first case we can write

$$
\begin{aligned}
& \sum_{i=L+1}^{L+\left\lfloor\kappa_{n} s\right\rfloor}\left(m_{(1)}\left(\boldsymbol{X}_{i}\right)-\bar{m}_{n}\left(\boldsymbol{X}_{i}\right)\right) \omega_{n}\left(\boldsymbol{X}_{i}\right) I\left\{\boldsymbol{X}_{i} \leq \boldsymbol{z}\right\} \\
& =\sum_{i=L+1}^{L+\left\lfloor\kappa_{n} s\right\rfloor}\left(m_{(1)}\left(\boldsymbol{X}_{i}\right)-\frac{m_{(1)}\left(\boldsymbol{X}_{i}\right) \sum_{j=1}^{\left\lfloor n s_{0}\right\rfloor} f_{j}\left(\boldsymbol{X}_{i}\right)}{\sum_{j=1}^{n} f_{j}\left(\boldsymbol{X}_{i}\right)}-\frac{m_{(2)}\left(\boldsymbol{X}_{i}\right) \sum_{j=\left\lfloor n s_{0}\right\rfloor+1}^{n} f_{j}\left(\boldsymbol{X}_{i}\right)}{\sum_{j=1}^{n} f_{j}\left(\boldsymbol{X}_{i}\right)}\right) \\
& \quad \omega_{n}\left(\boldsymbol{X}_{i}\right) I\left\{\boldsymbol{X}_{i} \leq \boldsymbol{z}\right\} \\
& =\sum_{i=L+1}^{L+\left\lfloor\kappa_{n} s\right\rfloor}\left(m_{(1)}\left(\boldsymbol{X}_{i}\right)-m_{(2)}\left(\boldsymbol{X}_{i}\right)\right) \frac{\sum_{j=\left\lfloor n s_{0}\right\rfloor+1}^{n} f_{j}\left(\boldsymbol{X}_{i}\right)}{\sum_{j=1}^{n} f_{j}\left(\boldsymbol{X}_{i}\right)} \omega_{n}\left(\boldsymbol{X}_{i}\right) I\left\{\boldsymbol{X}_{i} \leq \boldsymbol{z}\right\}
\end{aligned}
$$


and analogously for the second case

$$
\begin{aligned}
& \sum_{i=L \vee\left\lfloor n s_{0}\right\rfloor+1}^{L+\left\lfloor\kappa_{n} s\right\rfloor}\left(m_{(2)}\left(\boldsymbol{X}_{i}\right)-\bar{m}_{n}\left(\boldsymbol{X}_{i}\right)\right) \omega_{n}\left(\boldsymbol{X}_{i}\right) I\left\{\boldsymbol{X}_{i} \leq \boldsymbol{z}\right\} \\
= & \sum_{i=L \vee\left\lfloor n s_{0}\right\rfloor+1}^{L+\left\lfloor\kappa_{n} s\right\rfloor}\left(m_{(2)}\left(\boldsymbol{X}_{i}\right)-m_{(1)}\left(\boldsymbol{X}_{i}\right)\right) \frac{\sum_{j=1}^{\left\lfloor n s_{0}\right\rfloor} f_{j}\left(\boldsymbol{X}_{i}\right)}{\sum_{j=1}^{n} f_{j}\left(\boldsymbol{X}_{i}\right)} \omega_{n}\left(\boldsymbol{X}_{i}\right) I\left\{\boldsymbol{X}_{i} \leq \boldsymbol{z}\right\} .
\end{aligned}
$$

We will only examine the case $L+\left\lfloor\kappa_{n} s\right\rfloor \leq\left\lfloor n s_{0}\right\rfloor$ in detail since the other case works analogously.

The remainder of the proof is similar to the proof of Lemma A.2. With $g\left(\boldsymbol{X}_{i}\right):=$ $\left(m_{(1)}\left(\boldsymbol{X}_{i}\right)-m_{(2)}\left(\boldsymbol{X}_{i}\right)\right)$ and $\bar{f}_{n}^{\left(s_{0}\right)}\left(\boldsymbol{X}_{i}\right)=\frac{\sum_{j=\left\lfloor n s_{0}\right\rfloor+1}^{n} f_{j}\left(\boldsymbol{X}_{i}\right)}{\sum_{j=1}^{n} f_{j}\left(\boldsymbol{X}_{i}\right)}$ it holds

$$
\begin{aligned}
& \sup _{s \in[0,1]} \sup _{z \in \mathbb{R}^{d}}\left|\sum_{i=L+1}^{L+\left\lfloor\kappa_{n} s\right\rfloor} g\left(\boldsymbol{X}_{i}\right) I\left\{\left|g\left(\boldsymbol{X}_{i}\right)\right|>\kappa_{n}^{\frac{1}{r}}\right\} \bar{f}_{n}^{\left(s_{0}\right)}\left(\boldsymbol{X}_{i}\right) \omega_{n}\left(\boldsymbol{X}_{i}\right) I\left\{\boldsymbol{X}_{i} \leq \boldsymbol{z}\right\}\right| \\
& \leq \sum_{i=L+1}^{L+\kappa_{n}}\left|g\left(\boldsymbol{X}_{i}\right)\right| I\left\{\left|g\left(\boldsymbol{X}_{i}\right)\right|>\kappa_{n}^{\frac{1}{r}}\right\}
\end{aligned}
$$

and further

$$
P\left(\sum_{i=L+1}^{L+\kappa_{n}}\left|g\left(\boldsymbol{X}_{i}\right)\right| I\left\{\left|g\left(\boldsymbol{X}_{i}\right)\right|>\kappa_{n}^{\frac{1}{r}}\right\}>C \kappa_{n}\right) \leq C^{-1} \kappa_{n}^{-1} C_{m} \kappa_{n} \kappa_{n}^{\frac{1}{r}-1}
$$

by the Markov inequality with

$$
\begin{aligned}
E\left[\left|g\left(\boldsymbol{X}_{i}\right)\right| I\left\{\left|g\left(\boldsymbol{X}_{i}\right)\right|>\kappa_{n}^{\frac{1}{r}}\right\}\right] & =E\left[\left|g\left(\boldsymbol{X}_{i}\right)\right|^{r}\left|g\left(\boldsymbol{X}_{i}\right)\right|^{-(r-1)} I\left\{\left|g\left(\boldsymbol{X}_{i}\right)\right|>\kappa_{n}^{\frac{1}{r}}\right\}\right] \\
& \leq \kappa_{n}^{-\frac{r-1}{r}} E\left[\left|g\left(\boldsymbol{X}_{i}\right)\right|^{r}\right] \\
& \leq C_{m} \kappa_{n}^{\frac{1}{r}-1}
\end{aligned}
$$

for all $i$ and for some $C_{m}<\infty$ by assumption (II). Thus we can rewrite our assertion as

$$
P\left(\sup _{s \in[0,1]} \sup _{\varphi \in \mathcal{F}_{n}}\left|\left(\sum_{i=L+1}^{L+\left\lfloor\kappa_{n} s\right\rfloor} \varphi\left(\boldsymbol{X}_{i}\right)-\int \varphi d P\right)\right|>C \kappa_{n}\right) \leq \bar{C} \kappa_{n}^{\frac{1}{r}-1}
$$

with the function class

$$
\mathcal{F}_{n}:=\left\{\boldsymbol{x} \mapsto g(\boldsymbol{x}) I\left\{|g(\boldsymbol{x})| \leq \kappa_{n}^{\frac{1}{r}}\right\} \bar{f}_{n}^{\left(s_{0}\right)}(\boldsymbol{x}) \omega_{n}(\boldsymbol{x}) I\{\boldsymbol{x} \leq \boldsymbol{z}\}: \boldsymbol{z} \in \mathbb{R}^{d}\right\}
$$


To replace the supremum over $\varphi$ by a maximum we cover $\mathcal{F}_{n}$ by finitely many brackets $\left[\varphi_{j}^{l}, \varphi_{j}^{u}\right]_{j \in \times_{i=1}^{d}\left\{1, \ldots, N_{i}\right\}}$ where

$$
\begin{aligned}
\varphi_{\boldsymbol{j}}^{u}(\boldsymbol{x}):= & g(\boldsymbol{x}) I\left\{|g(\boldsymbol{x})| \leq \kappa_{n}^{\frac{1}{r}}\right\} I\{g(\boldsymbol{x}) \geq 0\} \bar{f}_{n}^{\left(s_{0}\right)}(\boldsymbol{x}) \omega_{n}(\boldsymbol{x}) I\left\{\boldsymbol{x} \leq \boldsymbol{z}_{\boldsymbol{j}}\right\} \\
& +g(\boldsymbol{x}) I\left\{|g(\boldsymbol{x})| \leq \kappa_{n}^{\frac{1}{r}}\right\} I\{g(\boldsymbol{x})<0\} \bar{f}_{n}^{\left(s_{0}\right)}(\boldsymbol{x}) \omega_{n}(\boldsymbol{x}) I\left\{\boldsymbol{x} \leq \boldsymbol{z}_{\boldsymbol{j}-\mathbf{1}}\right\}
\end{aligned}
$$

and

$$
\begin{aligned}
\varphi_{\boldsymbol{j}}^{u}(\boldsymbol{x}):= & g(\boldsymbol{x}) I\left\{|g(\boldsymbol{x})| \leq \kappa_{n}^{\frac{1}{r}}\right\} I\{g(\boldsymbol{x}) \geq 0\} \bar{f}_{n}^{\left(s_{0}\right)}(\boldsymbol{x}) \omega_{n}(\boldsymbol{x}) I\left\{\boldsymbol{x} \leq \boldsymbol{z}_{j-1}\right\} \\
& +g(\boldsymbol{x}) I\left\{|g(\boldsymbol{x})| \leq \kappa_{n}^{\frac{1}{r}}\right\} I\{g(\boldsymbol{x})<0\} \bar{f}_{n}^{\left(s_{0}\right)}(\boldsymbol{x}) \omega_{n}(\boldsymbol{x}) I\left\{\boldsymbol{x} \leq \boldsymbol{z}_{j}\right\}
\end{aligned}
$$

and $\boldsymbol{j}, z_{j}$ are defined as in the proof of Lemma A.2. The total number of brackets $J_{n}:=N_{[]}\left(\epsilon_{n}, \mathcal{F}_{n},\|\cdot\|_{L_{1}(P)}\right)$ needed to cover $\mathcal{F}_{n}$ is again of order $J_{n}=O\left(\epsilon_{n}^{-d}\right)$, which follows analogously to but easier than the proof of Lemma A.7 in Mohr (2018). Now we proceed completely analogously to the proof of Lemma A.2 by replacing the supremum over $s$ by a maximum as well and applying Liebscher's Theorem. Since the arguments are the same as in the aforementioned proof we omit this part for the sake of brevity.

Lemma A.4 Under the assumptions of Theorem 3.1 as well as under those of Theorem 4.1 it holds

$P\left(\sup _{s \in[0,1]} \sup _{z \in \mathbb{R}^{d}}\left|\sum_{i=L+1}^{L+\left\lfloor\kappa_{n} s\right\rfloor}\left(\bar{m}_{n}\left(\boldsymbol{X}_{i}\right)-\hat{m}_{n}\left(\boldsymbol{X}_{i}\right)\right) \omega_{n}\left(\boldsymbol{X}_{i}\right) I\left\{\boldsymbol{X}_{i} \leq \boldsymbol{z}\right\}\right|>C \kappa_{n}\right) \leq C^{-1} \kappa_{n}^{-\zeta}$

for all $L=0,1, \ldots, n-\kappa_{n}, 1 \leq \kappa_{n} \leq n, n \in \mathbb{N}$ and all $C>0$ with $\zeta>0$ from assumption (VIII).

Proof of Lemma A.4 It holds

$$
\begin{aligned}
& P\left(\sup _{s \in[0,1]} \sup _{z \in \mathbb{R}^{d}}\left|\sum_{i=L+1}^{L+\left\lfloor\kappa_{n} s\right\rfloor}\left(\bar{m}_{n}\left(\boldsymbol{X}_{i}\right)-\hat{m}_{n}\left(\boldsymbol{X}_{i}\right)\right) \omega_{n}\left(\boldsymbol{X}_{i}\right) I\left\{\boldsymbol{X}_{i} \leq \boldsymbol{z}\right\}\right|>C \kappa_{n}\right) \\
& \leq P\left(\sum_{i=L+1}^{L+\kappa_{n}}\left|\bar{m}_{n}\left(\boldsymbol{X}_{i}\right)-\hat{m}_{n}\left(\boldsymbol{X}_{i}\right)\right| \omega_{n}\left(\boldsymbol{X}_{i}\right)>C \kappa_{n}\right) \\
& \leq P\left(\sup _{\boldsymbol{x} \in \boldsymbol{J}_{n}}\left|\bar{m}_{n}(\boldsymbol{x})-\hat{m}_{n}(\boldsymbol{x})\right|>C\right) \\
& \leq C^{-1} E\left[\sup _{\boldsymbol{x} \in \boldsymbol{J}_{n}}\left|\bar{m}_{n}(\boldsymbol{x})-\hat{m}_{n}(\boldsymbol{x})\right|\right]
\end{aligned}
$$


by the Markov inequality. Further by Lemma A.1 with assumption (VIII) it holds that

$$
\frac{\sup _{\boldsymbol{x} \in \boldsymbol{J}_{n}}\left|\bar{m}_{n}(\boldsymbol{x})-\hat{m}_{n}(\boldsymbol{x})\right|}{n^{-\zeta}} \underset{n \rightarrow \infty}{\stackrel{P}{\longrightarrow}} 0
$$

which implies

$$
\frac{E\left[\sup _{\boldsymbol{x} \in \boldsymbol{J}_{n}}\left|\bar{m}_{n}(\boldsymbol{x})-\hat{m}_{n}(\boldsymbol{x})\right|\right]}{n^{-\zeta}} \underset{n \rightarrow \infty}{\longrightarrow} 0
$$

and thus for sufficiently large $n$

$$
\begin{aligned}
E\left[\sup _{\boldsymbol{x} \in \boldsymbol{J}_{n}}\left|\bar{m}_{n}(\boldsymbol{x})-\hat{m}_{n}(\boldsymbol{x})\right|\right] & \leq n^{-\zeta} \\
& \leq \kappa_{n}^{-\zeta}
\end{aligned}
$$

for $\kappa_{n} \leq n$. This completes the proof.

\section{A.2 Proof of main results}

We will proof Theorem 3.1 under the assumption (IX.1) and simply make a note on the parts that change under (IX.2).

Proof of Theorem 3.1 First note that for all $s \in[0,1]$ and $z \in \mathbb{R}^{d}$

$$
\hat{T}_{n}(s, z)=A_{n}(s, z)+\Delta_{n, 1}(s) \Delta_{n, 2}(z),
$$

where $A_{n}(s, z)=A_{n, 1}(s, z)+A_{n, 2}(s, z)+A_{n, 3}(s, z)+A_{n, 4}(s, z)$ with

$$
\begin{aligned}
& A_{n, 1}(s, \boldsymbol{z}):= \frac{1}{n} \sum_{i=1}^{\lfloor n s\rfloor} U_{i} \omega_{n}\left(\boldsymbol{X}_{i}\right) I\left\{\boldsymbol{X}_{i} \leq \boldsymbol{z}\right\} \\
& A_{n, 2}(s, \boldsymbol{z}):=\frac{1}{n} \sum_{i=1}^{\left\lfloor n\left(s \wedge s_{0}\right)\right\rfloor}\left(\left(m_{(1)}\left(\boldsymbol{X}_{i}\right)-\bar{m}_{n}\left(\boldsymbol{X}_{i}\right)\right) \omega_{n}\left(\boldsymbol{X}_{i}\right) I\left\{\boldsymbol{X}_{i} \leq \boldsymbol{z}\right\}\right. \\
&\left.-E\left[\left(m_{(1)}\left(\boldsymbol{X}_{i}\right)-\bar{m}_{n}\left(\boldsymbol{X}_{i}\right)\right) \omega_{n}\left(\boldsymbol{X}_{i}\right) I\left\{\boldsymbol{X}_{i} \leq \boldsymbol{z}\right\}\right]\right) \\
& A_{n, 3}(s, \boldsymbol{z}):=I\left\{s>s_{0}\right\} \frac{1}{n} \sum_{i=\left\lfloor n s_{0}\right\rfloor+1}^{\lfloor n s\rfloor}\left(\left(m_{(2)}\left(\boldsymbol{X}_{i}\right)-\bar{m}_{n}\left(\boldsymbol{X}_{i}\right)\right) \omega_{n}\left(\boldsymbol{X}_{i}\right) I\left\{\boldsymbol{X}_{i} \leq \boldsymbol{z}\right\}\right. \\
&\left.-E\left[\left(m_{(2)}\left(\boldsymbol{X}_{i}\right)-\bar{m}_{n}\left(\boldsymbol{X}_{i}\right)\right) \omega_{n}\left(\boldsymbol{X}_{i}\right) I\left\{\boldsymbol{X}_{i} \leq \boldsymbol{z}\right\}\right]\right) \\
& A_{n, 4}(s, \boldsymbol{z}):=\frac{1}{n} \sum_{i=1}^{\lfloor n s\rfloor}\left(\bar{m}_{n}\left(\boldsymbol{X}_{i}\right)-\hat{m}_{n}\left(\boldsymbol{X}_{i}\right)\right) \omega_{n}\left(\boldsymbol{X}_{i}\right) I\left\{\boldsymbol{X}_{i} \leq \boldsymbol{z}\right\}
\end{aligned}
$$


and

$$
\begin{aligned}
& \Delta_{n, 1}(s):=I\left\{s \leq s_{0}\right\} \frac{n-\left\lfloor n s_{0}\right\rfloor}{n} \frac{\lfloor n s\rfloor}{n}+I\left\{s>s_{0}\right\} \frac{n-\lfloor n s\rfloor}{n} \frac{\left\lfloor n s_{0}\right\rfloor}{n} \\
& \Delta_{n, 2}(\boldsymbol{z}):=\int_{(-\infty, z]}\left(m_{(1)}(\boldsymbol{x})-m_{(2)}(\boldsymbol{x})\right) f(\boldsymbol{x}) \omega_{n}(\boldsymbol{x}) d \boldsymbol{x},
\end{aligned}
$$

since by inserting the definition of $\bar{m}_{n}$ we obtain for $s \leq s_{0}$

$$
\begin{aligned}
& \frac{1}{n} \sum_{i=1}^{\lfloor n s\rfloor} E\left[\left(m_{(1)}\left(\boldsymbol{X}_{i}\right)-\bar{m}_{n}\left(\boldsymbol{X}_{i}\right)\right) \omega_{n}\left(\boldsymbol{X}_{i}\right) I\left\{\boldsymbol{X}_{i} \leq \boldsymbol{z}\right\}\right] \\
& \quad=\frac{n-\left\lfloor n s_{0}\right\rfloor}{n} \frac{1}{n} \sum_{i=1}^{\lfloor n s\rfloor} E\left[\left(m_{(1)}\left(\boldsymbol{X}_{i}\right)-m_{(2)}\left(\boldsymbol{X}_{i}\right)\right) \omega_{n}\left(\boldsymbol{X}_{i}\right) I\left\{\boldsymbol{X}_{i} \leq \boldsymbol{z}\right\}\right] \\
& \quad=\frac{n-\left\lfloor n s_{0}\right\rfloor}{n} \frac{\lfloor n s\rfloor}{n} \Delta_{n, 2}(\boldsymbol{z})
\end{aligned}
$$

and for $s>s_{0}$

$$
\begin{aligned}
\frac{1}{n} \sum_{i=1}^{\left\lfloor n s_{0}\right\rfloor} E\left[\left(m_{(1)}\left(\boldsymbol{X}_{i}\right)-\bar{m}_{n}\left(\boldsymbol{X}_{i}\right)\right) \omega_{n}\left(\boldsymbol{X}_{i}\right) I\left\{\boldsymbol{X}_{i} \leq \boldsymbol{z}\right\}\right] \\
+\frac{1}{n} \sum_{i=\left\lfloor n s_{0}\right\rfloor+1}^{\lfloor n s\rfloor} E\left[\left(m_{(2)}\left(\boldsymbol{X}_{i}\right)-\bar{m}_{n}\left(\boldsymbol{X}_{i}\right)\right) \omega_{n}\left(\boldsymbol{X}_{i}\right) I\left\{\boldsymbol{X}_{i} \leq \boldsymbol{z}\right\}\right] \\
=\frac{n-\left\lfloor n s_{0}\right\rfloor}{n} \frac{1}{n} \sum_{i=1}^{\left\lfloor n s_{0}\right\rfloor} E\left[\left(m_{(1)}\left(\boldsymbol{X}_{i}\right)-m_{(2)}\left(\boldsymbol{X}_{i}\right)\right) \omega_{n}\left(\boldsymbol{X}_{i}\right) I\left\{\boldsymbol{X}_{i} \leq \boldsymbol{z}\right\}\right] \\
\quad-\frac{\left\lfloor n s_{0}\right\rfloor}{n} \frac{1}{n} \sum_{i=\left\lfloor n s_{0}\right\rfloor+1}^{\lfloor n s\rfloor} E\left[\left(m_{(1)}\left(\boldsymbol{X}_{i}\right)-m_{(2)}\left(\boldsymbol{X}_{i}\right)\right) \omega_{n}\left(\boldsymbol{X}_{i}\right) I\left\{\boldsymbol{X}_{i} \leq \boldsymbol{z}\right\}\right] \\
=\frac{n-\lfloor n s\rfloor}{n} \frac{\left\lfloor n s_{0}\right\rfloor}{n} \Delta_{n, 2}(\boldsymbol{z}) .
\end{aligned}
$$

Note that we use the notation $\int_{(\infty, z]} g(\boldsymbol{x}) d \boldsymbol{x}=\int_{-\infty}^{z_{d}} \ldots \int_{-\infty}^{z_{1}} g\left(x_{1}, \ldots, x_{d}\right) d x_{1} \ldots d x_{d}$ here. Due to the dominated convergence theorem and assumption (II), it holds that

$$
\Delta_{n, 1}(s) \Delta_{n, 2}(z)=\Delta_{1}(s) \Delta_{2}(z)+o(1)
$$

uniformly in $s \in[0,1]$ and $z \in \mathbb{R}^{d}$, where

$$
\begin{aligned}
& \Delta_{1}(s):=I\left\{s \leq s_{0}\right\}\left(1-s_{0}\right) s+I\left\{s>s_{0}\right\}(1-s) s_{0}, \\
& \Delta_{2}(\boldsymbol{z}):=\int_{(-\infty, z]}\left(m_{(1)}(\boldsymbol{x})-m_{(2)}(\boldsymbol{x})\right) f(\boldsymbol{x}) d \boldsymbol{x} .
\end{aligned}
$$


Note that under (IX.2) the same assertion holds with

$$
\Delta_{n, 2}(z):=\int_{(-\infty, z]}\left(m_{(1)}(\boldsymbol{x})-m_{(2)}(\boldsymbol{x})\right) \frac{f_{(1)}(\boldsymbol{x}) f_{(2)}(\boldsymbol{x})}{\frac{\left\lfloor n s_{0}\right\rfloor}{n} f_{(1)}(\boldsymbol{x})+\frac{n-\left\lfloor n s_{0}\right\rfloor}{n} f_{(2)}(\boldsymbol{x})} \omega_{n}(\boldsymbol{x}) d \boldsymbol{x}
$$

and

$$
\Delta_{2}(z):=\int_{(-\infty, z]}\left(m_{(1)}(\boldsymbol{x})-m_{(2)}(\boldsymbol{x})\right) \frac{f_{(1)}(\boldsymbol{x}) f_{(2)}(\boldsymbol{x})}{s_{0} f_{(1)}(\boldsymbol{x})+\left(1-s_{0}\right) f_{(2)}(\boldsymbol{x})} d \boldsymbol{x} .
$$

By Lemmata A.2, A.3 and A.4 with $\kappa_{n}=n$, it holds that $A_{n}(s, z)=o_{P}(1)$ uniformly in $s \in[0,1]$ and $z \in \mathbb{R}^{d}$. Hence, we have shown that

$$
\sup _{z \in \mathbb{R}^{d}}\left|\hat{T}_{n}(s, z)\right|=\Delta_{1}(s) \sup _{z \in \mathbb{R}^{d}}\left|\Delta_{2}(z)\right|+o_{P}(1)
$$

uniformly in $s \in[0,1]$ under both cases (IX.1) and (IX.2). The assertion then follows by Theorem 2.12 in Kosorok (2008) as $s_{0}$ is well-separated maximum of $[0,1] \rightarrow$ $\mathbb{R}, s \mapsto \Delta_{1}(s)$.

Remark Note that there are examples of $m_{(1)}, m_{(2)}$ and $f$ resp. $f_{(1)}, f_{(2)}$ that lead to $\Delta_{2}(\infty)=0$. In those cases a change point estimator based on the classical CUSUM $\hat{T}_{n}(s, \infty)$ is not consistent.

Proof of Theorem 3.2 First note that $s_{0}=\frac{\left\lfloor n s_{0}\right\rfloor}{n}+O\left(n^{-1}\right)$ and $\hat{s}_{n}=\frac{\left\lfloor n \hat{s}_{n}\right\rfloor}{n}$. Thus we can consider $\left|\frac{\left\lfloor n \hat{s}_{n}\right\rfloor}{n}-\frac{\left\lfloor n s_{0}\right\rfloor}{n}\right|$ instead of $\left|\hat{s}_{n}-s_{0}\right|$. The proof follows mainly along the same lines as the proof of Theorem 1 in Hariz et al. (2007). Consider the norm $N: l^{\infty}\left(\mathbb{R}^{d}\right) \rightarrow \mathbb{R}, g \mapsto \sup _{z \in \mathbb{R}^{d}}|g(z)|$ and let $M>0$. We will show below that for all $\eta>0$ and $b, c>0$ it holds

$$
\begin{aligned}
P\left(r_{n}\left|\frac{\left\lfloor n \hat{s}_{n}\right\rfloor}{n}-\frac{\left\lfloor n s_{0}\right\rfloor}{n}\right|>2^{M}\right)= & P\left(r_{n}^{-1} 2^{M}<\left|\frac{\left\lfloor n \hat{s}_{n}\right\rfloor}{n}-\frac{\left\lfloor n s_{0}\right\rfloor}{n}\right| \leq \eta\right) \\
& +P\left(\left|\frac{\left\lfloor n \hat{s}_{n}\right\rfloor}{n}-\frac{\left\lfloor n s_{0}\right\rfloor}{n}\right|>\eta\right) \\
\leq & E_{n, 1}+E_{n, 2}+E_{n, 3}+E_{n, 4}
\end{aligned}
$$

where

$$
\begin{aligned}
E_{n, 1}:= & P\left(r_{n}^{-1} 2^{M}<\left|\frac{\left\lfloor n \hat{s}_{n}\right\rfloor}{n}-\frac{\left\lfloor n s_{0}\right\rfloor}{n}\right| \leq \eta\right. \\
& \left.N\left(A_{n}\left(\hat{s}_{n}, \cdot\right)-A_{n}\left(s_{0}, \cdot\right)\right) \geq C\left|\frac{\left\lfloor n \hat{s}_{n}\right\rfloor}{n}-\frac{\left\lfloor n s_{0}\right\rfloor}{n}\right|\right) \\
E_{n, 2}:= & P\left(N\left(A_{n}\left(s_{0}, \cdot\right)\right)>c\right)
\end{aligned}
$$




$$
\begin{aligned}
& E_{n, 3}:=P\left(\Delta_{n, 1}\left(s_{0}\right) N\left(\Delta_{n, 2}(\cdot)\right) \leq b\right) \\
& E_{n, 4}:=P\left(\left|\hat{s}_{n}-s_{0}\right|>\eta\right),
\end{aligned}
$$

with $C:=b-2 c$. Now it holds that $E_{n, 4} \rightarrow 0$ for all $\eta>0$, due to Theorem 3.1. Further, $E_{n, 2} \rightarrow 0$ for all $c>0$ as $A_{n}\left(s_{0}, z\right)=o_{P}(1)$ holds uniformly in $z \in \mathbb{R}^{d}$. Finally choose $b>0$ and $n^{\prime}=n^{\prime}(b) \in \mathbb{N}$ such that $E_{n, 3}=0$ for all $n \geq n^{\prime}$, which exists as $\Delta_{1}\left(s_{0}\right) N\left(\Delta_{2}(\cdot)\right)>0$ and $\Delta_{n, 1}\left(s_{0}\right) N\left(\Delta_{n, 2}(\cdot)\right)=\Delta_{1}\left(s_{0}\right) N\left(\Delta_{2}(\cdot)\right)+o(1)$. We then choose $c>0$ such that $b-2 c>0$. To see the validity of (A.9) first note that for all $s \in[0,1]$

$$
\begin{aligned}
\hat{T}_{n}(s, \cdot) & =A_{n}(s, \cdot)+\Delta_{n, 1}(s) \Delta_{n, 2}(\cdot) \\
& =A_{n}(s, \cdot)-A_{n}\left(s_{0}, \cdot\right)+A_{n}\left(s_{0}, \cdot\right)\left(1-\frac{\Delta_{n, 1}(s)}{\Delta_{n, 1}\left(s_{0}\right)}\right)+\frac{\Delta_{n, 1}(s)}{\Delta_{n, 1}\left(s_{0}\right)} \hat{T}_{n}\left(s_{0}, \cdot\right) .
\end{aligned}
$$

Applying the norm and triangular inequality we obtain for all $s \in[0,1]$

$$
\begin{aligned}
N\left(\hat{T}_{n}(s, \cdot)\right) \leq & N\left(A_{n}(s, \cdot)-A_{n}\left(s_{0}, \cdot\right)\right)+\left(1-\frac{\Delta_{n, 1}(s)}{\Delta_{n, 1}\left(s_{0}\right)}\right) N\left(A_{n}\left(s_{0}, \cdot\right)\right) \\
& +\left(\frac{\Delta_{n, 1}(s)}{\Delta_{n, 1}\left(s_{0}\right)}\right) N\left(\hat{T}_{n}\left(s_{0}, \cdot\right)\right)
\end{aligned}
$$

which is equivalent to

$$
\begin{aligned}
N\left(\hat{T}_{n}(s, \cdot)\right)-N\left(\hat{T}_{n}\left(s_{0}, \cdot\right)\right) \leq & N\left(A_{n}(s, \cdot)-A_{n}\left(s_{0}, \cdot\right)\right) \\
& +\left(\frac{\Delta_{n, 1}(s)}{\Delta_{n, 1}\left(s_{0}\right)}-1\right)\left(N\left(\hat{T}_{n}\left(s_{0}, \cdot\right)\right)-N\left(A_{n}\left(s_{0}, \cdot\right)\right)\right) .
\end{aligned}
$$

Due to the definition of $\hat{s}_{n}$ it holds that $N\left(\hat{T}_{n}\left(\hat{s}_{n}, \cdot\right)\right)-N\left(\hat{T}_{n}\left(s_{0}, \cdot\right)\right) \geq 0$. Additionally using the specific definition of $\Delta_{n, 1}$ we obtain

$$
\begin{aligned}
& N\left(A_{n}\left(\hat{s}_{n}, \cdot\right)-A_{n}\left(s_{0}, \cdot\right)\right) \\
& \quad \geq\left(1-\frac{\Delta_{n, 1}\left(\hat{s}_{n}\right)}{\Delta_{n, 1}\left(s_{0}\right)}\right)\left(N\left(\hat{T}_{n}\left(s_{0}, \cdot\right)\right)-N\left(A_{n}\left(s_{0}, \cdot\right)\right)\right) \\
& \quad \geq \underbrace{\min \left(\frac{n}{\left\lfloor n s_{0}\right\rfloor}, \frac{n}{n-\left\lfloor n s_{0}\right\rfloor}\right)}_{>1}\left|\frac{\left\lfloor n \hat{s}_{n}\right\rfloor}{n}-\frac{\left\lfloor n s_{0}\right\rfloor}{n}\right|\left(N\left(\hat{T}_{n}\left(s_{0}, \cdot\right)\right)-N\left(A_{n}\left(s_{0}, \cdot\right)\right)\right) \\
& \geq\left|\frac{\left\lfloor n \hat{s}_{n}\right\rfloor}{n}-\frac{\left\lfloor n s_{0}\right\rfloor}{n}\right|\left(\Delta_{n, 1}\left(s_{0}\right) N\left(\Delta_{n, 2}(\cdot)\right)-2 N\left(A_{n}\left(s_{0}, \cdot\right)\right)\right),
\end{aligned}
$$


where we again make use of the triangular inequality in the last step. Putting the results together we obtain

$$
\begin{aligned}
& P\left(r_{n}^{-1} 2^{M}<\left|\frac{\left\lfloor n \hat{s}_{n}\right\rfloor}{n}-\frac{\left\lfloor n s_{0}\right\rfloor}{n}\right| \leq \eta\right) \\
& \leq P\left(r_{n}^{-1} 2^{M}<\left|\frac{\left\lfloor n \hat{s}_{n}\right\rfloor}{n}-\frac{\left\lfloor n s_{0}\right\rfloor}{n}\right| \leq \eta, \Delta_{n, 1}\left(s_{0}\right) N\left(\Delta_{n, 2}(\cdot)\right)>b, N\left(A_{n}\left(s_{0}, \cdot\right)\right) \leq c\right) \\
&+P\left(\Delta_{n, 1}\left(s_{0}\right) N\left(\Delta_{n, 2}(\cdot)\right) \leq b\right)+P\left(N\left(A_{n}\left(s_{0}, \cdot\right)\right)>c\right) \\
& \leq P\left(r_{n}^{-1} 2^{M}<\left|\frac{\left\lfloor n \hat{s}_{n}\right\rfloor}{n}-\frac{\left\lfloor n s_{0}\right\rfloor}{n}\right| \leq \eta,\right. \\
&\left.N\left(A_{n}\left(\hat{s}_{n}, \cdot\right)-A_{n}\left(s_{0}, \cdot\right)\right) \geq C\left|\frac{\left\lfloor n \hat{s}_{n}\right\rfloor}{n}-\frac{\left\lfloor n s_{0}\right\rfloor}{n}\right|\right) \\
&+P\left(\Delta_{n, 1}\left(s_{0}\right) N\left(\Delta_{n, 2}(\cdot)\right) \leq b\right)+P\left(N\left(A_{n}\left(s_{0}, \cdot\right)\right)>c\right) .
\end{aligned}
$$

Finally we will investigate $E_{n, 1}$. To do this we define shells

$$
S_{n, l}=\left\{t \in[0,1]: 2^{l}<r_{n}\left|t-\frac{\left\lfloor n s_{0}\right\rfloor}{n}\right| \leq 2^{l+1}\right\}
$$

and choose $L_{n}=L_{n}(\eta)$ such that $2^{L_{n}}<r_{n} \eta \leq 2^{L_{n}+1}$ for some $\eta \leq \frac{1}{2}$. Then

$$
\begin{aligned}
E_{n, 1} & \leq \sum_{l=M}^{L_{n}} P\left(\frac{\left\lfloor n \hat{s}_{n}\right\rfloor}{n} \in S_{n, l}, N\left(A_{n}\left(\hat{s}_{n}, \cdot\right)-A_{n}\left(s_{0}, \cdot\right)\right) \geq C\left|\frac{\left\lfloor n \hat{s}_{n}\right\rfloor}{n}-\frac{\left\lfloor n s_{0}\right\rfloor}{n}\right|\right) \\
& \leq \sum_{l=M}^{L_{n}} P\left(\sup _{s:\left\lfloor\frac{\lfloor n s\rfloor}{n}-\frac{\left\lfloor n s_{0}\right\rfloor}{n} \mid \leq 2^{l+1} r_{n}^{-1}\right.} N\left(A_{n}(s, \cdot)-A_{n}\left(s_{0}, \cdot\right)\right) \geq C 2^{l} r_{n}^{-1}\right) \\
& \leq \sum_{l=M}^{L_{n}} \sum_{i=1}^{4} P\left(\sup _{s:\left\lfloor\frac{\lfloor n s\rfloor}{n}-\frac{\left\lfloor n s_{0}\right\rfloor}{n} \mid \leq 2^{l+1} r_{n}^{-1}\right.} N\left(A_{n, i}(s, \cdot)-A_{n, i}\left(s_{0}, \cdot\right)\right) \geq \frac{C}{4} 2^{l} r_{n}^{-1}\right) \\
& \leq \tilde{C}\left(\left(\frac{n}{r_{n}}\right)^{\frac{1}{q}-1} \sum_{l=M}^{L_{n}}\left(2^{\frac{1}{q}-1}\right)^{l}+\left(\frac{n}{r_{n}}\right)^{\frac{1}{r}-1} \sum_{l=M}^{L_{n}}\left(2^{\frac{1}{r}-1}\right)^{l}+\left(\frac{n}{r_{n}}\right)^{-\zeta} \sum_{l=M}^{L_{n}}\left(2^{-\zeta}\right)^{l}\right)
\end{aligned}
$$

for some constant $\tilde{C}<\infty$ by Lemmata A.2, A.3 and A.4 with $\kappa_{n}=\left\lfloor 2^{l+1} \frac{n}{r_{n}}\right\rfloor$ with $q$ from assumption (I), $r$ from assumption (II) and $\zeta>0$ from assumption (VIII). Now choosing $r_{n}=n$ and letting $n$ and thus $L_{n}$ tend to infinity and then $M$ to infinity, the assertion of Theorem 3.2 follows. 
Proof of Theorem 4.1 Under (IX.3) we have for all $s \in[0,1]$ and $z \in \mathbb{R}$

$$
\hat{T}_{n}(s, z)=A_{n}(s, z)+\Delta_{n, 1}(s) \Delta_{n, 2}(z)+\tilde{\Delta}_{n}(s, z),
$$

with $A_{n}(s, z)$ and $\Delta_{n, 1}(s)$ from the proof of Theorem 3.1, and with

$$
\begin{aligned}
& \Delta_{n, 2}(z) \\
& :=\int_{(-\infty, z]}\left(m_{(1)}(x)-m_{(2)}(x)\right) \frac{f_{(1)}(x) f_{(2)}(x)}{\frac{\left\lfloor n s_{0}\right\rfloor}{n} f_{(1)}(x)+\frac{n-\left\lfloor n s_{0}\right\rfloor}{n} f_{(2)}(x)+R_{n}(x)} \omega_{n}(x) d x
\end{aligned}
$$

and

$$
\begin{aligned}
\tilde{\Delta}_{n}(s, z) & \\
:= & \int_{(-\infty, z]}\left(m_{(1)}(x)-m_{(2)}(x)\right) I\left\{s \leq s_{0}\right\} \frac{\lfloor n s\rfloor}{n} \\
& \cdot \frac{f_{(1)}(x) R_{n}(x)}{\frac{\left\lfloor n s_{0}\right\rfloor}{n} f_{(1)}(x)+\frac{n-\left\lfloor n s_{0}\right\rfloor}{n} f_{(2)}(x)+R_{n}(x)} \omega_{n}(x) d x .
\end{aligned}
$$

Now it holds that

$$
\Delta_{n, 2}(z) \rightarrow \int_{(-\infty, z]}\left(m_{(1)}(x)-m_{(2)}(x)\right) \frac{f_{(1)}(x) f_{(2)}(x)}{s_{0} f_{(1)}(x)+\left(1-s_{0}\right) f_{(2)}(x)} d x=: \Delta_{2}(z)
$$

and $\tilde{\Delta}_{n}(s, z) \rightarrow 0$ uniformly in $s \in[0,1]$ and $z \in \mathbb{R}$, due to dominated convergence and assumption (II). Hence we have uniformly in $s$ and $z$

$$
\hat{T}_{n}(s, z)=A_{n}(s, z)+\Delta_{1}(s) \Delta_{2}(z)+o(1),
$$

with $\Delta_{1}(s)$ as in the proof of Theorem 3.1. The rest goes analogously to the proof of Theorem 3.1.

Remark Note that for finite $n \in \mathbb{N}$ we do not get the decomposition of $\hat{T}_{n}$ as in (A.4) in the proof of Theorem 3.1. We only obtain this kind of decomposition when letting $n$ tend to infinity. The decomposition for finite $n$, however, is essential for the proof of the rates of convergence in Theorem 3.2.

\section{References}

Antoch J, Hušková M, Prášková Z (1997) Effect of dependence on statistics for determination of change. J Stat Plan Inference 60:291-310

Aue A, Horváth L, Hušková M (2012) Segmenting mean-nonstationary time series via trending regressions. J Econ 168:367-381

Bai J (1994) Least squares estimation of a shift in linear processes. J Time Ser Anal 15:435-472

Bai J (1997) Estimation of a change point in multiple regression models. Rev Econ Stat 79:551-563

Chen G, Choi YK, Zhou Y (2005) Nonparametric estimation of structural change points in volatility models for time series. J Econ 126:79-114 
Chong TT-L (2001) Structural change in AR(1) models. Econ Theory 17:87-155

Delgado MA, Hidalgo J (2000) Nonparametric inference on structural breaks. J Econ 96:113-144

Döring M, Jensen U (2015) Smooth change point estimation in regression models with random design. Ann Inst Stat Math 67:595-619

Eichinger B, Kirch C (2018) A MOSUM procedure for the estimation of multiple random change points. Bernoulli 24:526-564

Ferger D (2005) Weighted least squares estimators for a change-point. Econ Qual Control 20:255-270

Ferger D, Stute W (1992) Convergence of changepoint estimators. Stoch Process Appl 42:345-351

Franke J, Kreiss J-P, Mammen E, Neumann M (2002) Properties of the nonparametric autoregressive bootstrap. J Time Ser Anal 23:555-585

Fryzlewicz P (2014) Wild binary segmentation for multiple change-point detection. Ann Stat 42:2243-2281

Fryzlewicz P (2019) Detecting possibly frequent change-points: wild binary segmentation 2 and steepestdrop model selection. Preprint on arXiv:1812.06880

Hariz SB, Wylie JJ, Zhang Q (2007) Optimal rate of convergence for nonparametric change-point estimators for nonstationary sequences. Ann Stat 35:1802-1826

Horváth L, Hušková M, Serbinowska M (1997) Estimators for the time of change in linear models. Statistics 29:109-130

Hušková M, Antoch J (2003) Detection of structural changes in regression. Tatra Mt Math Publ 26:201-215

Hušková M, Kirch C (2008) Bootstrapping confidence intervals for the change? Point of time series. J Time Ser Anal 29:947-972

Kirch C, Kamgaing JT (2012) Testing for parameter stability in nonlinear autoregressive models. J Time Ser Anal 33:365-385

Kirch C, Muhsal B, Ombao H (2015) Detection of changes in multivariate time series with application to EEG data. J Am Stat Assoc 110:1197-1216

Kosorok MR (2008) Introduction to empirical processes and semiparametric inference. Springer, New York

Kristensen D (2009) Uniform convergence rates of kernel estimators with heterogeneous dependent data. Econ Theory 25:1433-1445

Kristensen D (2012) Non-parametric detection and estimation of structural change. Econ J 15:420-461

Liebscher E (1996) Strong convergence of sums of $\alpha$-mixing random variables with applications to density estimation. Stoch Process. Appl. 65:69-80

Mohr M (2018) Changepoint detection in a nonparametric time series regression model. PhD thesis, University of Hamburg. http://ediss.sub.uni-hamburg.de/volltexte/2018/9416/

Mohr M, Neumeyer N (2019) Consistent nonparametric change point detection combining CUSUM and marked empirical processes. Preprint on arXiv:1901.08491

Pang T, Zhang D (2015) Asymptotic inferences for an AR(1) model with a change point and possibly infinite variance. Commun Stat Theory Methods 44:4848-4865

Pang T, Zhang D, Chong TT-L (2014) Asymptotic inferences for an AR(1) model with a change point: stationary and nearly non-stationary cases. J Time Ser Anal 35:133-150

Publisher's Note Springer Nature remains neutral with regard to jurisdictional claims in published maps and institutional affiliations. 